An Evaluation of a Structured

Overlapping Grid Implementation of FCT for High-Speed Flows

J. W. Banks, J. N. Shadid

August 26, 2011

Flux-Corrected-Transport 
This document was prepared as an account of work sponsored by an agency of the United States government. Neither the United States government nor Lawrence Livermore National Security, LLC, nor any of their employees makes any warranty, expressed or implied, or assumes any legal liability or responsibility for the accuracy, completeness, or usefulness of any information, apparatus, product, or process disclosed, or represents that its use would not infringe privately owned rights. Reference herein to any specific commercial product, process, or service by trade name, trademark, manufacturer, or otherwise does not necessarily constitute or imply its endorsement, recommendation, or favoring by the United States government or Lawrence Livermore National Security, LLC. The views and opinions of authors expressed herein do not necessarily state or reflect those of the United States government or Lawrence Livermore National Security, LLC, and shall not be used for advertising or product endorsement purposes. 


\section{Contents}

An Evaluation of a Structured Overlapping Grid Implementation of

FCT for High-Speed Flows ${ }^{*} \ldots \ldots \ldots \ldots \ldots \ldots \ldots \ldots \ldots \ldots \ldots \ldots \ldots$

J. W. Banks and J. N. Shadid

$1 \quad$ Introduction .................................. 4

2 Governing Equations $\ldots \ldots \ldots \ldots \ldots \ldots \ldots \ldots \ldots \ldots \ldots \ldots \ldots$

3 Flux-Corrected-Transport Algorithm $\ldots \ldots \ldots \ldots \ldots \ldots \ldots \ldots$

$3.1 \quad$ Overlapping grids and AMR $\ldots \ldots \ldots \ldots \ldots \ldots \ldots \ldots$

3.2 FCT Discretization on a mapped grid ............. 7

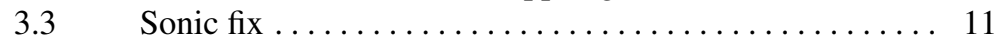

$3.4 \quad$ Strong Rarefactions ......................... 13

3.5 A Note Concerning Monotonicity ................. 14

$4 \quad$ Numerical Results............................... 15

4.1 Method of analytic solutions ................. 15

4.2 Isolated Contact and Shock Discontinuities .......... 18

$4.3 \quad$ Sod's shock tube problem (modified) .............. 19

4.4 A Two-Shock Riemann Problem ................. 21

4.5 Shu-Osher Problem ......................... 22

4.6 Shock Impingement on Stationary Cylinder. . . . . . . . . 25

4.7 Irregular Mach Reflection of a Strong Shock ......... 30

4.8 Shock impingement on a collection of cylinders ....... 34

$4.9 \quad$ An Idealized Z-pinch Implosion . . . . . . . . . . . . . . 37

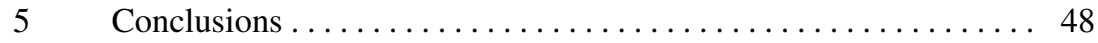

References ................................. 49 



\title{
An Evaluation of a Structured Overlapping Grid Implementation of FCT for High-Speed Flows*
}

\author{
J. W. Banks and J. N. Shadid
}

\begin{abstract}
This study considers the development and assessment of a Flux-Corrected Transport (FCT) algorithm for simulating high-speed flows on structured overlapping grids. This class of algorithm shows promise for solving some difficult highlynonlinear problems where robustness and control of certain features, such as maintaining positive densities, is important. Complex, possibly moving, geometry is treated through the use of structured overlapping grids. Adaptive mesh refinement (AMR) is employed to ensure sharp resolution of discontinuities in an efficient manner. Improvements to the FCT algorithm are proposed for the treatment of strong rarefaction waves as well as rarefaction waves containing a sonic point. Simulation results are obtained for a set of test problems and the convergence characteristics are demonstrated and compared to a high-resolution Godunov method. The problems considered include smooth manufactured solutions, isolated shock and contact discontinuities, a modified Sod shock-tube problem, a two-shock Riemann problem, the Shu-Osher test problem, shock impingement on single cylinder, irregular Mach reflection of a strong shock striking an inclined plane, shock impingement on multiple fixed and movable cylinders, and an idealized Z-pinch implosion problem.

${ }^{*}$ This work was partially supported by the DOE Office of Science, Advanced Scientific Computing Research-Applied Mathematics Program at Sandia National Laboratory under contract DE-AC04-94AL85000 and at Lawrence Livermore National Laboratory under Contract DE-AC52-07NA27344.
\end{abstract}

\footnotetext{
J. W. Banks

Center for Applied Scientific Computing

Lawrence Livermore National Laboratory

Livermore, CA

e-mail: banks20@1lnl.gov

J. N. Shadid

Computational Sciences Research Institute

Sandia National Laboratories

Albuquerque, NM

e-mail: jnshadi@sandia.gov
} 


\section{Introduction}

Many physical systems are well described mathematically by systems of conservation laws. Typical examples might include fluid flow around a body, condensed phase explosives, astrophysical phenomenon, or high energy density physics applications. A large number of such systems have the property that discontinuous solutions can arise in finite time even from smooth initial data. These discontinuities can be of a linear (e.g. contact wave) or nonlinear form (e.g. shock wave). Numerical methods need to balance the often competing requirements of accurately approximating these two types of discontinuities while at the same time requiring higher order accuracy in smooth regions of the solution. This balance has been one of the primary drivers behind the development of modern simulation tools. Methods striking such a balance are often referred to as high-resolution methods and they require the use of limiters (switches) that choose between a number of different numerical stencils. For some flow regimes, this type of limiting has been found to be essential to obtain robust schemes. Many, if not most, of the high-resolution techniques have their roots in the 1970's with ideas originally developed by Boris and Book in connection with flux-corrected transport (FCT) $[11,12,13]$. In the intervening years, FCT has been applied to a wide range of challenging applications, for examples see [14, 56, 39]. Recently, the developments of Kuzmin et. al. [39] towards algebraic flux correction as well as implicit methods have produced a renewed interest in FCT as a useful numerical method for many applications.

The scope of the current study is to review work performed in $[6,8]$ in extending the FCT method for compressible flow simulations into the context of overlapping grids which are used to represent geometric complexities as well as ensure mesh regularity $[17,31,32,7]$. The overlapping grid method is quite general and can be used to generate computational meshes for complex geometries $[3,16,46]$ without the use of unstructured meshes, cut cells for embedded boundaries, or overly contorted globally mapped grids. FCT has not been used extensively for overlapping grids and the current study brings together some of the work in that field.

This work examines implementation details for FCT applied to structured overlapping grids. We include a discussion about the extension of FCT to moving overlapping grids. A series of test problems demonstrates the properties of the method for practical simulations and compares the results with those from a high-resolution Godunov method. Reference to the results presented in other studies such as [24, 48] gives a good understanding of the relative merits of these various high-resolution shock capturing schemes. This comparison is particularly useful for cases where Riemann solution based strategies are not viable because of the complexities of the governing equations. Such is the case for some relativistic flows, for example, and the FCT method may be attractive in this context provided an appropriate low order method can be devised without resort to Riemann solutions. As in [8], FCT can also be useful for problems with extreme jumps in density and pressure where traditional high-resolution methods may fail due to unphysical states such as negative densities. We overview this work and present the prototype Z-pinch implosion model as well as simulation results from the FCT method. 
The remainder of the chapter is structured as follows. In Section 2 the governing equations are presented. In Section 3 the FCT algorithm is presented and the development for structured overlapping grids is summarized. This section also presents a brief discussion of two open issues with the traditional FCT algorithm; that of performance when either strong or sonic rarefactions are present in the flow. The poor performance of the standard method for these problems is demonstrated and an improvement of the algorithm is proposed and evaluated. Section 4 presents numerical results for the FCT method and provides a comparison to a high-resolution Godunov method. Some qualitative remarks concerning computational cost comparisons between the FCT and Godunov methods are presented in Section ?? and concluding remarks are given in Section 5.

\section{Governing Equations}

We consider the flow of an inviscid compressible gas and assume that in two dimensions the density $\rho$, velocities $\left(u_{1}, u_{2}\right)$, pressure $p$, and total energy $E$ satisfy the system of conservation laws

$$
\frac{\partial}{\partial t} \mathbf{u}+\frac{\partial}{\partial x_{1}} \mathbf{f}_{1}(\mathbf{u})+\frac{\partial}{\partial x_{2}} \mathbf{f}_{2}(\mathbf{u})=0
$$

where

$$
\mathbf{u}=\left[\begin{array}{c}
\rho \\
\rho u_{1} \\
\rho u_{2} \\
E
\end{array}\right] \quad \mathbf{f}_{1}(\mathbf{u})=\left[\begin{array}{c}
\rho u_{1} \\
\rho u_{1}^{2}+p \\
\rho u_{1} u_{2} \\
u_{1}(E+p)
\end{array}\right] \quad \mathbf{f}_{2}(\mathbf{u})=\left[\begin{array}{c}
\rho u_{2} \\
\rho u_{1} u_{2} \\
\rho u_{2}^{2}+p \\
u_{2}(E+p)
\end{array}\right] .
$$

System (1) defines the conservation of mass, momenta, and total energy for the gas and is recognized as the well known compressible Euler equations in two space dimensions. In this formulation, the total energy is given by

$$
E=\rho e+\frac{1}{2} \rho\left(u_{1}^{2}+u_{2}^{2}\right)
$$

where $e=e(\rho, p)$ is the specific internal energy, which is specified by an equation of state. This paper assumes an ideal equation of state, namely

$$
e=\frac{p}{\rho(\gamma-1)}
$$

where $\gamma=\frac{c_{p}}{c_{v}}$ is the ratio of (constant) specific heats with $c_{p}$ the specific heat at constant pressure and $c_{v}$ the specific heat at constant volume. The Euler equations (1) are assumed to have been non-dimensionalized with suitable reference quantities and all results are presented in dimensionless units. 


\section{Flux-Corrected-Transport Algorithm}

This section describes the FCT method as used in this chapter including the extensions and modifications we have made to the classic FCT algorithm. This implementation includes a DeVore type pre-limiter in lieu of Zalesak's flux pre-constraint, removal of artificial diffusion prior to the FCT flux limiter, a Jameson style artificial viscosity, a sonic fix for entropy violating rarefaction waves, and the extension of the FCT algorithm to overlapping grids. For clarity, the improvements for treating sonic points and very strong rarefactions are left to the end of the section.

\subsection{Overlapping grids and AMR}

We consider the governing equations (1) and proceed with a description of the FCT method in a two dimensional overlapping grid framework. To this end, we assume the flow domain is given by $\Omega$ and is discretized using an overlapping grid $\mathscr{G}$. The overlapping grid consists of a set of component grids $\left\{\mathscr{G}_{i}\right\}, i=1, \ldots, \mathscr{N}_{g}$, that cover $\Omega$ and overlap where they meet. Each component grid covers a sub-domain $\Omega_{i}$. Grid points are tagged as discretization points where the governing equations are applied, ghost points used for the application of boundary conditions, interpolation points where solution values are communicated between grids, or unused points where no computation is performed which are cut out through the mesh generation procedure. The FCT stencil is 7-points wide requiring three layers of data at interpolation and physical boundaries. At interpolation boundaries, the 7-point stencil would normally require three layers of interpolation points. Although we can generate such grids, in practice we usually construct a grid with a single layer of interpolation points and obtain values at the two additional layers through extrapolation. At physical boundaries, values on the boundary and three layers of ghost points are obtained through application of the physical boundary conditions, derived compatibility conditions, and extrapolation following the approach described in [31, 32]. Note that the dependence of the solution on this final extrapolated layer is extremely weak as it can only affect whether the chosen update at the boundary is first or second order accurate (i.e. it is used only in the determination of the $\alpha$ in (8) below. For more details concerning general overlapping grid methods, including application of boundary conditions, see $[17,31,32,30]$. Adaptive mesh refinement (AMR) is used in regions of the flow where the solution changes rapidly, such as near shocks and contact surfaces. We employ a block-structured AMR approach following that described originally in [10] and using modifications for overlapping grids as presented in $[31,32,7]$. 


\subsection{FCT Discretization on a mapped grid}

Each component grid, including base-level grids and any refined grids, is defined by a mapping from the unit square in computational space $\left(r_{1}, r_{2}\right)$ to physical space $\left(x_{1}, x_{2}\right)$. In computational space, equation (1) becomes

$$
\frac{\partial}{\partial t} \mathbf{u}+\frac{1}{J} \frac{\partial}{\partial r_{1}} \mathbf{F}_{1}(\mathbf{u})+\frac{1}{J} \frac{\partial}{\partial r_{2}} \mathbf{F}_{2}(\mathbf{u})=0
$$

where

$$
\mathbf{F}_{1}(\mathbf{u})=J\left(\frac{\partial r_{1}}{\partial x_{1}} \mathbf{f}_{1}+\frac{\partial r_{1}}{\partial x_{2}} \mathbf{f}_{2}\right), \quad \mathbf{F}_{2}(\mathbf{u})=J\left(\frac{\partial r_{2}}{\partial x_{1}} \mathbf{f}_{1}+\frac{\partial r_{2}}{\partial x_{2}} \mathbf{f}_{2}\right)
$$

and

$$
J=\left|\frac{\partial\left(x_{1}, x_{2}\right)}{\partial\left(r_{1}, r_{2}\right)}\right| .
$$

The metrics of the mapping, $\partial x_{1} / \partial r_{2}, \partial x_{2} / \partial r_{2}$, etc., and the Jacobian are considered to be known for each component grid at the time of computation and can be generated analytically or approximated.

Discretization of (3) is performed using a uniform grid $\left(r_{1, i}, r_{2, j}\right)$ with grid spacing $\left(\Delta r_{1}, \Delta r_{2}\right)$. The FCT method is generally considered a two-step process proceeding first with a low order update and finishing with the high-resolution FCT correction. We begin with the formulation of the low order solution update

$$
\mathbf{u}_{i, j}^{\mathrm{td}, n}=\mathbf{u}_{i, j}^{n}-\frac{\Delta t}{J_{i, j} \Delta r_{1}} D_{+r_{1}} \mathbf{F}_{1}^{\text {low }, n}-\frac{\Delta t}{J_{i, j} \Delta r_{2}} D_{+r_{2}} \mathbf{F}_{2, j, j-1 / 2}^{\text {low }, n}
$$

where $D_{+r_{1}}$ and $D_{+r_{2}}$ are the undivided forward difference approximations in the $r_{1}$ and $r_{2}$ directions of index space respectively. The "td" notation is consistent with $[11,13,12,59]$ and denotes "transported and diffused". For this work the HLL low order flux $[27,55]$ is used and for curvilinear geometries is given by

$\mathbf{F}_{1+1 / 2, j}^{\text {low }, n}=\left\{\begin{array}{lll}\mathbf{F}_{1 i, j}^{n} & \text { if } & s_{-} \geq 0 \\ \mathbf{F}_{1 i+1, j}^{n} & \text { if } & s_{+} \leq 0 \\ \frac{s_{+}}{s_{+}-s_{-}} \mathbf{F}_{1 i, j}^{n}-\frac{s_{-}}{s_{+}-s_{-}} \mathbf{F}_{1+1, j}^{n}+\frac{s_{-} s_{+}}{s_{+}-s_{-}} D_{+r_{1}} \mathbf{u}_{i, j}^{n} & \text { else }\end{array}\right.$

where

$$
\begin{aligned}
& s_{-}=\min \left(v_{i, j}^{n}-c_{i, j}^{n}, v_{i+1, j}^{n}-c_{i+1, j}^{n}\right)\left\|\left(\frac{\partial r_{1}}{\partial x_{1}}, \frac{\partial r_{1}}{\partial x_{2}}\right)\right\|_{2}, \\
& s_{+}=\max \left(v_{i, j}^{n}+c_{i, j}^{n}, v_{i+1, j}^{n}+c_{i+1, j}^{n}\right)\left\|\left(\frac{\partial r_{1}}{\partial x_{1}}, \frac{\partial r_{1}}{\partial x_{2}}\right)\right\|_{2},
\end{aligned}
$$

$c_{i, j}^{n}$ is the sound speed in a given cell, and $v_{i, j}^{n}$ is the component of the velocity normal to the cell face. The fluxes across other cell boundaries take similar forms. 
It should be noted that in $[60,39]$, Zalesak suggests the use of the Rusanov flux for the low order method. This is a symmetrized version of the HLL flux resulting in further diffusion than the original HLL flux. However, the Rusanov flux as presented in $[60,39]$ is slightly flawed in that the selected wave speed is not sufficient to encompass the full Riemann solution for all cases. A more general Rusanov flux is

$$
\mathbf{F}_{1_{i+1 / 2, j}}^{\text {low }, n}=\frac{1}{2}\left[\left(\mathbf{F}_{1+1, j}^{n}+\mathbf{F}_{1, j}^{n}\right)-\max \left(\left|\lambda_{i+1, j}^{n}\right|,\left|\lambda_{i, j}^{n}\right|\right) D_{+r_{1}} \mathbf{u}_{i, j}^{n}\right]
$$

where $\lambda_{i, j}^{n}$ is the largest eigenvalue (in magnitude) of the Jacobian matrix $\frac{\partial}{\partial \mathbf{u}} \mathbf{F}_{1}$ at a cell $(i, j)$ and time $t_{n}$. The difference between (6) and the equation presented in $[60,39]$ is the use of $\max \left(\left|\lambda_{i+1, j}^{n}\right|,\left|\lambda_{i, j}^{n}\right|\right)$ rather than $1 / 2\left(\left|\lambda_{i+1, j}^{n}\right|+\left|\lambda_{i, j}^{n}\right|\right)$. In this work, the HLL flux is used but we have found that the Rusanov flux (6) works nearly as well and is less expensive. As presented, both of these approximate fluxes require knowledge of the eigenvalues of the Jacobian matrix. If this information were not known, a Lax-Friedrichs type flux could in principle be used instead.

The second step of the FCT algorithm requires an "anti-diffusive" flux which is defined as the difference between a high-order flux and the low-order one. In the $r_{1}$ direction of index space for example, this is

$$
\mathbf{F}_{1 \pm 1 / 2, j}^{\mathrm{AD}, n}=\mathbf{F}_{1_{i \pm 1 / 2, j}}^{\mathrm{high}, n}-\mathbf{F}_{1_{i \pm 1 / 2, j}}^{\text {low }, n}
$$

The high order flux is typically chosen to be some high-order centered flux and for this work the centered second-order flux

$$
\mathbf{F}_{1_{i+1 / 2, j}}^{\operatorname{high}_{i+n}}=\frac{1}{2}\left(\mathbf{F}_{1, j}^{n}+\mathbf{F}_{1+1, j}^{n}\right)
$$

is chosen. The final sub-step update is now defined as

$\mathbf{u}_{i, j}^{\text {new }}=\mathbf{u}_{i, j}^{\mathrm{td}, n}-\frac{\Delta t}{J_{i, j} \Delta r_{1}} D_{+r_{1}}\left(\alpha_{i-1 / 2, j}^{n} \odot \mathbf{F}_{1_{i-1 / 2, j}}^{\mathrm{AD}, n}\right)-\frac{\Delta t}{J_{i, j} \Delta r_{2}} D_{+r_{2}}\left(\alpha_{i, j-1 / 2}^{n} \odot \mathbf{F}_{2, j-1 / 2}^{\mathrm{AD}, n}\right)$

where $\odot$ indicates component-wise multiplication. The vector of $\alpha$ 's are chosen using the FCT algorithm as described below and represent the proportion of antidiffusive flux at each cell face that is used in the final update. Our choice of notation facilitates the use of the FCT algorithm in a method of lines type approach. By defining

$$
\frac{\partial}{\partial t} \mathbf{u}_{i, j}^{n}=\frac{\mathbf{u}_{i, j}^{\text {new }}-\mathbf{u}_{i, j}^{n}}{\Delta t}
$$

we obtain an updated solution $\mathbf{u}_{i, j}^{n+1}$ using any ordinary differential equation (ODE) integrator we choose. Choices for ODE integrators might include Runge-Kutta methods, Adams methods, or others. For this work, we use an explicit Adams predictor-corrector method of second order to match the spatial algorithm. Detail concerning the implementation of these time integrators can be found for example in $[2,30]$. 
Consider the determination of $\alpha_{i+1 / 2, j}^{n}$. FCT seeks to enforce solution monotonicity through the choice of $\alpha$, but the property of monotonicity is valid only for characteristic variables [57]. For the non-linear Euler equations, conversion to characteristic variables requires both a linearization and an eigen-decomposition of the linearized problem. As such, we linearize about the arithmetic average $\overline{\mathbf{u}}=$ $\frac{1}{2}\left(\mathbf{u}_{i, j}^{\mathrm{td}, n}+\mathbf{u}_{i+1, j}^{\mathrm{td}, n}\right)$. More sophisticated choices, such as the Roe average [55], could be made but in our experience these make little difference in the eventual computed solutions. From this state, the linearized eigen-decomposition $\mathbf{T}^{-1} \Lambda \mathbf{T}=\mathbf{A}=\frac{\partial}{\partial \mathbf{u}} \mathbf{F}_{1}(\overline{\mathbf{u}})$ is found where we have dropped the sub- and superscripts to simplify the exposition. Whenever multiplication by $\mathbf{T}$ is performed to achieve characteristic quantities it should be understood that this implies linearization about a particular face, in this case $(i+1 / 2, j)$. For two dimensions, a large number of characteristic transformations must be performed (in three dimensions the number is even larger) and this constitutes one of the most expensive parts of the FCT method.

In [14], DeVore indicates that the scheme of Zalesak does not preserve monotonicity in two dimensions and suggests limiting the fluxes using the original Boris/Book limiter $[11,13]$ in each direction prior to their input to the multidimensional limiter. This is straight forward to and we demonstrate it for $\mathbf{F}_{1_{i+1 / 2, j}} \mathrm{AD}_{, n}$

$\hat{\mathbf{F}}_{1 i+1 / 2, j}^{\mathrm{AD}, n}=\mathbf{s} \odot \max \left[0, \min \left(\left|\mathbf{T F}_{1_{i+1 / 2, j}}^{\mathrm{AD}, n}\right|, \mathbf{s} \odot \frac{J_{i+1 / 2, j} \Delta r_{1}}{\Delta t} D_{+r_{1}} \mathbf{T u}_{i+1 / 2, j}^{\mathrm{td}, n}, \mathbf{s} \odot \frac{J_{i+1 / 2, j} \Delta r_{1}}{\Delta t} D_{+r_{1}} \mathbf{T u}_{i-1 / 2, j}^{\mathrm{td}, n}\right)\right]$,

where $\mathbf{s}=\operatorname{sign}\left(\mathbf{T F}_{1_{i+1 / 2, j}}^{\mathrm{AD}, n}\right)$ and the "hat" notation indicates that the anti-diffusive flux has been pre-limited. The other $\hat{\mathbf{F}}$ fluxes are obtained through similar formulas.

To complete the FCT algorithm, define the local maximum and minimum characteristic values as

$$
\begin{aligned}
& \mathbf{w}_{k}^{\max }=\max \left(\mathbf{T u}_{i+k-1, j}^{\mathrm{td}, n}, \mathbf{T u}_{i+k, j}^{\mathrm{td}, n}, \mathbf{T u}_{i+k+1, j}^{\mathrm{td}, n}, \mathbf{T u}_{i+k, j-1}^{\mathrm{td}, n}, \mathbf{T u}_{i+k, j+1}^{\mathrm{td}, n}\right), \\
& \mathbf{w}_{k}^{\min }=\min \left(\mathbf{T u}_{i+k-1, j}^{\mathrm{td}, n}, \mathbf{T u}_{i+k, j}^{\mathrm{td}, n}, \mathbf{T u}_{i+k+1, j}^{\mathrm{td}, n}, \mathbf{T u}_{i+k, j-1}^{\mathrm{td}, n}, \mathbf{T u}_{i+k, j+1}^{\mathrm{td}, n}\right),
\end{aligned}
$$

where $k=0,1$ and the extrema are taken component-wise. The actual influx into the cells on either side of the cell face which would result from the AD fluxes is computed for example as

$$
\begin{aligned}
\mathbf{I}_{k}= & \frac{1}{\Delta r_{1}}\left[\max \left(\frac{\hat{\mathbf{F}}_{1 i+k-1 / 2, j}}{\mathrm{AD}_{i+k, j}}, 0\right)-\min \left(\frac{\hat{\mathbf{F}}_{1+k+1 / 2, j}}{\mathrm{AD}_{i+k, j}}, 0\right)\right] \\
& +\frac{1}{\Delta r_{2}}\left[\max \left(\frac{\hat{\mathbf{F}}_{2 i+k, j-1 / 2}}{\mathrm{AD}_{i+k, j}}, 0\right)-\min \left(\frac{\hat{\mathbf{F}}_{2 i+k, j+1 / 2}}{J_{i+k, j}}, 0\right)\right],
\end{aligned}
$$

and the maximum permissible influx such that the characteristic bounds from (10) are not violated, indicated by the tilde, is for example 


$$
\tilde{\mathbf{I}}_{k}=\frac{1}{\Delta t}\left[\mathbf{w}_{k}^{\max }-\mathbf{T u}_{i+k, j}^{\mathrm{td}, n}\right] .
$$

Notice in (11) that the influx into the cells from both direction of index space are considered simultaneously. This follows from [59] and reflects the fully multidimensional nature of this limiter as opposed to a limiter which is split along dimensional lines. Component-wise ratios of permissible to actual fluxes are then defined for the two cells as

$$
\mathbf{R}_{k}^{+}=\min \left(\frac{\tilde{\mathbf{I}}_{k}}{\mathbf{I}_{k}}, 1\right)
$$

The quantities $\mathbf{R}_{k}^{-}$, which represent the ratio of actual AD flux leaving the cell to the maximum flux permitted to leave the cell without violation of the bounds in (10), are defined using similar reasoning. Setting

$$
\begin{aligned}
\mathbf{O}_{k}= & \frac{1}{\Delta r_{1}}\left[\max \left(\frac{\hat{\mathbf{F}}_{1_{i+k+1 / 2, j}}^{\mathrm{AD}_{, n}}}{J_{i+k, j}}, 0\right)-\min \left(\frac{\hat{\mathbf{F}}_{1 i+k-1 / 2, j} \mathrm{AD}_{, n}}{J_{i+k, j}}, 0\right)\right] \\
& +\frac{1}{\Delta r_{2}}\left[\max \left(\frac{\hat{\mathbf{F}}_{2 i+k, j+1 / 2}}{\mathrm{AD}_{i+k, j}}, 0\right)-\min \left(\frac{\hat{\mathbf{F}}_{2 i+k, j-1 / 2}}{\mathrm{AD}_{i+k, j}}, 0\right)\right],
\end{aligned}
$$

and

$$
\tilde{\mathbf{O}}_{k}=\frac{1}{\Delta t}\left[\mathbf{T u}_{i+k, j}^{\mathrm{td}, n}-\mathbf{w}_{k}^{\min }\right]
$$

we define

$$
\mathbf{R}_{k}^{-}=\min \left(\frac{\tilde{\mathbf{O}}_{k}}{\mathbf{O}_{k}}, 1\right) .
$$

By choosing the most restrictive of these $\mathbf{R}$ values, the bounds from (10) are not violated. Thus we define

$$
\beta=\left\{\begin{array}{llc}
\min \left(\mathbf{R}_{0}^{+}, \mathbf{R}_{1}^{-}\right) & \text {when } & J_{i, j} \hat{\mathbf{F}}_{1+1 / 2, j}^{\mathrm{AD}, n}<0 \\
\min \left(\mathbf{R}_{1}^{+}, \mathbf{R}_{0}^{-}\right) & \text {when } & J_{i, j} \hat{\mathbf{F}}_{1+1 / 2, j}^{\mathrm{AD}, n} \geq 0 .
\end{array}\right.
$$

The final values for $\alpha_{i+1 / 2, j}^{n}$ are found through component-wise inversion of the formula

$$
\alpha_{i+1 / 2, j}^{n} \odot \mathbf{F}_{1_{i+1 / 2, j}}^{\mathrm{AD}_{, n}}=\mathbf{T}^{-1}\left(\beta \odot \hat{\mathbf{F}}_{1+1 / 2, j}^{\mathrm{AD}_{i+n}}\right) .
$$

It is important to note that monotonicity of the linearized characteristic variables does not imply monotonicity of the conserved variables. Thus the final updated solution could result in a negative density, imaginary sound speed, or negative pressure. Such events do occur in the simulations we present and must be treated in a rational and reasonable way. Zalesak suggests in $[59,39]$ that a fail-safe limiter be employed and we take a similar approach here. At each time, if the values at a given cell $(i, j)$ violate physically realistic bounds after advancement to $\mathbf{u}_{i, j}^{\text {new }}$ in (8), then no portion of the anti-diffusive flux is allowed at the boundaries of that cell. For such cells, 


$$
\alpha_{i+1 / 2, j}^{n}=\alpha_{i-1 / 2, j}^{n}=\alpha_{i, j+1 / 2}^{n}=\alpha_{i, j-1 / 2}^{n}=0
$$

is enforced and the method becomes fully first order in a local region. In our experience, this fail-safe mechanism is critical for the success of the FCT algorithm. It should also be noted that after setting $\alpha_{i \pm 1 / 2, j \pm 1 / 2}^{n}=0$ in one cell, the problem (negative density etc.) may then appear in a neighbouring cell. In principle the result could be a cascade across all cells. These cascades are rare and do not occur for any of the simulations presented in this work.

This completes the description of the FCT algorithm itself but there is another aspect which must be addressed. In [59] it is recognized that some amount of higher order dissipation must be included to remove high frequency noise generated by the FCT procedure. In that work the high-order dissipation was added to the AD flux prior to flux correction. In our studies we found this to be unsatisfactory because the effect of the high-order dissipation is reduced by the FCT limiters. The result is unacceptable levels of numerical noise in the computed solutions. Therefore we add dissipation independently after the FCT step. To this end we implement a secondorder dissipation near shocks $[19,31]$ to treat undamped transverse instabilities as well as a fourth-order Jameson style dissipation away from shocks [35, 34, 30]. We switch the fourth order dissipation on or off based on density variations to ensure that it is not active near shocks or contacts. One final note is that the computed solution will not violate the prescribed bounds only for CFL numbers less than $1 / 2$ and so all FCT simulation results presented in this chapter set the CFL number to be 0.4 .

\subsection{Sonic fix}

As is the case for some other methods, such as Godunov's method with an approximate Roe Riemann solver [55], the FCT method can exhibit poor behavior in rarefaction waves at points where the flow speed is equal to the sound speed (sonic points). The problem is illustrated by the solution to a modified version of Sod's shock tube problem $[52,55]$ with left and right states given by $\left(\rho, u_{1}, u_{2}, p\right)_{L}=$ $(1.0,0.75,0.0,1.0)$ and $\left(\rho, u_{1}, u_{2}, p\right)_{R}=(0.125,0.75,0.0,0.1)$, and with $\gamma=1.4$. We compute approximations to the solution of this Riemann problem using the grid $\mathscr{L}([-1,1], 100)$ where

$$
\mathscr{L}\left(\left[x_{a}, x_{b}\right], N\right)=\left\{x_{i} \mid x_{i}=x_{a}+i \Delta x, \Delta x=\left(x_{b}-x_{a}\right) / N, i=0,1, \ldots, N\right\},
$$

with the initial discontinuity located at $x=-0.4$. Figure 1 shows the results produced by the FCT method with and without our sonic fix. The problematic behavior of the method at the sonic point is clearly visible in the form of a rarefaction shock which represents an entropy violating weak solution.

The existence of rarefaction shocks in numerical approximations is typically the result of insufficient numerical diffusion. For FCT this is caused by the use of high- 

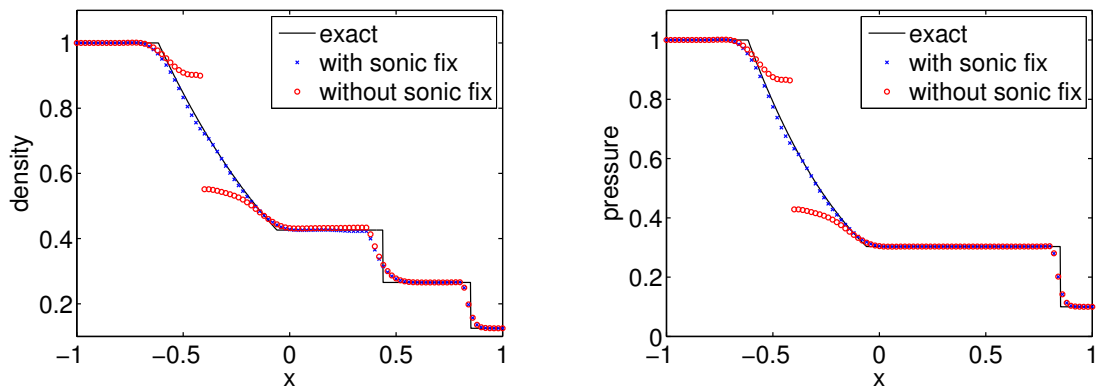

Fig. 1 FCT solution for a shock tube problem containing a sonic rarefaction with and without a sonic fix at $t=0.5$. The black line represents the exact solution, the red circles the numerical approximation without the sonic fix and the blue crosses the numerical approximation with the sonic fix. The problematic behavior at the sonic point is quite clear in both the density (left) and pressure (right).

order centered fluxes. This is in contrast to Roe's method where the linearization causes the problem even at first order. The FCT method considered in this chapter uses the HLL flux (known to be devoid of rarefaction shocks [55]) for the low order update. To eliminate rarefaction shocks for FCT approximations, we rely on this fact and simply set the value for $\alpha$ in (8) to zero for cases where sonic rarefactions are present. This choice has implications on solution accuracy, but because sonic points exist in isolation, the impact is negligible as will be demonstrated in Section 4.

The anti-diffusive fluxes in (8) have associated left and right states, call these $\mathbf{u}_{L}$ and $\mathbf{u}_{R}$ respectively. For instance consider $\mathbf{F}_{1_{i+1 / 2, j}}^{\mathrm{AD}, n}$ with $\mathbf{u}_{L}=\mathbf{u}_{i, j}^{\mathrm{td}, n}$ and $\mathbf{u}_{R}=\mathbf{u}_{i+1, j}^{\mathrm{td}, n}$. These states can be viewed as left and right states of a one dimensional Riemann problem in the direction normal to the cell face. Define the normal velocities as $v_{n, L}=\left(n_{1}, n_{2}\right) \cdot\left(u_{1 L}, u_{2 L}\right)^{T}$ and $v_{n, R}=\left(n_{1}, n_{2}\right) \cdot\left(u_{1 R}, u_{2 R}\right)^{T}$ where $\left(n_{1}, n_{2}\right)$ is the unit normal to the cell face. Following the nomenclature in [55], we define the star state as the center solution to this Riemann problem (i.e. the solution between the $\mathscr{C}^{+}$ and $\mathscr{C}^{-}$characteristics). As in [55], $p^{*}$ and $v_{n}^{*}$ can be approximated by

$$
p^{*}=\left[\max \left(0,\left(c_{L}+c_{R}-\frac{\gamma-1}{2}\left(v_{n, R}-v_{n, L}\right)\right)\left(\frac{c_{L}}{p_{L}^{z}}+\frac{c_{R}}{p_{R}^{z}}\right)^{-1}\right)\right]^{1 / z}
$$

and

$$
v_{n}^{*}=v_{n, L}+\frac{2}{\gamma-1}\left(c_{L}-c_{L}^{*}\right)
$$

where $c_{L}^{*}=c_{L}\left(p^{*} / p_{L}\right)^{z}, c_{R}^{*}=c_{R}\left(p^{*} / p_{R}\right)^{z}, z=(\gamma-1) /(2 \gamma), c_{L}$ is the left sound speed, and $c_{R}$ is the right sound speed. These particular star states arise from the approximation of the Riemann solution by the so-called two rarefaction Riemann solver and are approximations to the true star state. Note that other choices for the star states are also acceptable. Our sonic fix defines a new value for $\alpha$ by 


$$
\alpha_{i+1 / 2, j}^{n} \leftarrow\left\{\begin{array}{lll}
0 & \text { if } & v_{n, L}-c_{L} \leq 0 \text { and } v_{n}^{*}-c_{L}^{*} \geq 0 \\
0 & \text { if } & v_{n}^{*}+c_{R}^{*} \leq 0 \text { and } v_{n, R}+c_{R} \geq 0 \\
\alpha_{i+1 / 2, j}^{n} & \text { else. }
\end{array}\right.
$$

The effect of these choices is to return the solver to first order accuracy near sonic points in rarefaction waves. Figure 1 shows the solution to the modified Sod's problem employing this sonic fix where it is seen that the poor behavior has been effectively eliminated apart from a small kink at the sonic point. It should be noted that the particular sonic fix demonstrated here relies on an approximate solution to the Riemann problem. For cases where this solution is not known, this fix is not applicable and sonic rarefactions must be identified in another way. For example, one might consider applying the fix wherever the flow transitions from super- to sub-sonic flow across a cell boundary.

\subsection{Strong Rarefactions}

In addition to the poor behavior for sonic rarefaction waves, the traditional FCT algorithm runs into difficulties for strong rarefaction waves where the difference in velocities at which the gas is being pulled apart differ by more than the local sound speed. This is a very difficult problem for many methods because a near vacuum state is reached and failure can occur as a result of negative densities or pressures [56]. Consider the solution to a Riemann problem with left and right states $\left(\rho, u_{1}, u_{2}, p\right)_{L}=(1.0,-2.0,0.0,0.4)$ and $\left(\rho, u_{1}, u_{2}, p\right)_{L}=(1.0,2.0,0.0,0.4)$ respectively.

Figure 2 shows the density and velocity as computed by the FCT algorithm for this case both with and without our fix. The FCT solution without any fix demonstrates oscillations in velocity close to the near vacuum state (near the origin). In
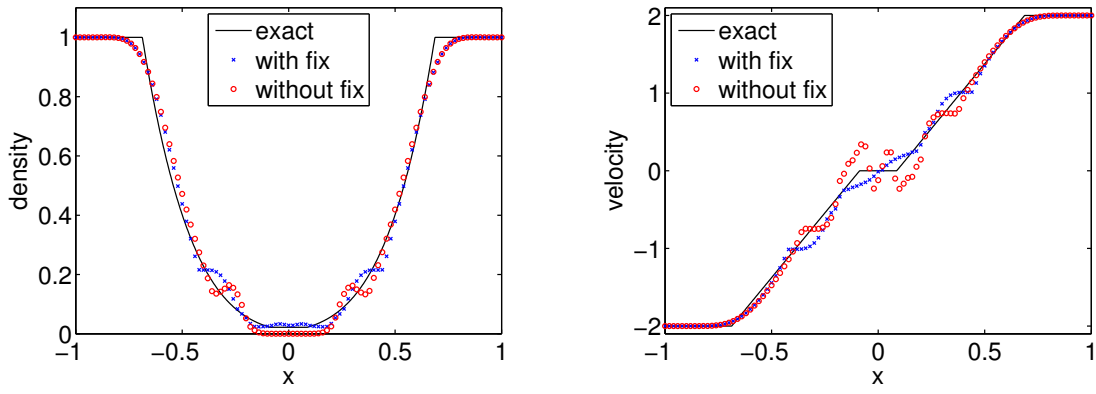

Fig. 2 Density (left) and velocity (right) for a strong rarefaction problem at $t=0.25$. The black line represents the exact solution, the red circles the FCT approximation without a fix and the blue crosses the FCT solution with the fix. The oscillations in velocity for the original FCT scheme are particularly troubling but also note the undershoot of the density near the origin. 
order to remove this behavior a simple fix is employed which sets

$$
\alpha_{i+1 / 2, j}^{n}=0 \quad \text { if } p^{*}<\min \left(p_{L}, p_{R}\right) \text { and }\left|v_{n_{L}}-v_{n_{R}}\right| \geq \max \left(c_{l}, c_{r}\right) .
$$

This causes the first order scheme to be used when strong rarefaction waves are present. The results shown in figure 2 demonstrate that the velocity from the fixed scheme is monotonic near the origin. These results are comparable to the results of Tóth in [56] but further improvements should be investigated.

\subsection{A Note Concerning Monotonicity}

The original FCT scheme of Boris and Book applied to 1-D linear advection problems is provably monotone. However, the extension by Zalesak to higherdimensions do not result in a monotone scheme, a fact that has apparently not been discussed in the literature. We now present a simple example to illustrate this fact. Consider linear advection with unit advection velocity,

$$
\frac{\partial}{\partial t} \rho+\frac{\partial}{\partial x_{1}} \rho=0
$$

We use the low-order flux given by $\mathbf{f}_{i+1 / 2}^{\text {low } n}=\rho_{i}^{n}$, and the second-order centered flux given by $\mathbf{f}_{i+1 / 2}^{\mathrm{high}, n}=\frac{1}{2}\left(\rho_{i}^{n}+\rho_{i+1}^{n}\right)$. At time level $t^{n}$ let the approximate solution be given by

$\rho_{-3}^{n}=4.5, \quad \rho_{-2}^{n}=4, \quad \rho_{-1}^{n}=3.5, \quad \rho_{0}^{n}=3, \quad \rho_{1}^{n}=3, \quad \rho_{2}^{n}=2, \quad \rho_{3}^{n}=1, \quad \rho_{4}^{n}=0$.

Set the grid spacing as $\Delta x_{1}=1$ and the temporal spacing as $\Delta t=0.25$. The FCT algorithm, as outlined by Zalesak [59, 60], produces the following values for $\alpha$

$$
\alpha_{-1 / 2}^{n}=1, \quad \alpha_{1 / 2}^{n}=1, \quad \alpha_{3 / 2}^{n}=1 .
$$

By using the forward Euler time integrator (i.e. $\rho_{i}^{n+1}=\rho_{i}^{\text {new }}$ ), the FCT solution after a single step results in the values

$$
\rho_{0}^{n+1}=3.0625, \quad \rho_{1}^{n+1}=3.125 .
$$

The solution at time $t^{n}$ was monotonically decreasing left to right while the solution for these two cells at time $t^{n+1}$ is monotonically increasing left to right and so the violation of monotonicity is demonstrated. Many authors suggest the use of a prelimiter, but for this case the pre-limiter suggested by Zalesak [59] and Kuzmin [39] has no effect as can be easily verified. The pre-limiter of DeVore [14], which we have adopted here, does remedy this particular problem, but a proof of monotonicity for arbitrary high order fluxes is not known. 


\section{Numerical Results}

We now present simulation results using the FCT algorithm described in Section 3. The discussion centers on studying the robustness and accuracy of the overall numerical approach as well as comparing the results to those from the high-resolution Godunov method in [31, 32, 7] which uses an approximate Roe Riemann solver [49] and the MinMod limiter [55]. Of course, any comparisons presented here are only valid for these particular implementations of the FCT and Godnuov methods. There are many variations to both algorithms which would change the specifics of the results. However, the present study provides a reasonable baseline comparison of the relative merits of the two schemes. Furthermore, the hope is that given the results from previous comparisons, for example in [24], one can place, in a general sense, high-resolution Godunov methods, WENO methods, and FCT in relation to each other. In fact the tests we present were largely driven by the choice of tests presented in [24] exactly for the reason that comparisons could be made.

Because the purpose of any comparisons made in this section is to provide a sense of the relative merits of the methods as they might be used in practice, the set of parameters used by each method is set to what we consider to be reasonable numbers. For the Godunov method we use $\mathrm{CFL}=0.9$ and for $\mathrm{FCT}$ we use $\mathrm{CFL}=0.4$. The small choice for FCT is required, as noted in Section 3, to ensure the desired bounds are not violated. For problems where AMR is used, the refinement criteria is the same for both schemes and is based on a weighted sum of first and second un-divided differences of the solution (see [31] for details).

We begin the discussion by establishing the expected second-order rate of convergence for the FCT method for smooth flows using the method of analytic solutions. Similar verification tests have also performed for the Godunov method as in [7], but direct comparisons are not made here because of the manufactured nature of the tests. Next we consider the solution to a series of problems including 1-D isolated contacts, isolated shocks, Sod's shock tube problem, a two-shock Riemann problem, and the Shu-Osher test case. The methods are then compared for the 2-D problems of shock impingement on a cylinder and the irregular Mach reflection of a strong shock on an inclined ramp. Finally, simulation results from the FCT method are presented for a number of more complex problems to include shock impingement on multiple fixed and moveable cylinders as well as for a prototype Z-pinch implosion problem. These problems are more difficult to characterize and so no comparison to the Godunov method is provided.

\subsection{Method of analytic solutions}

We now investigate convergence of the FCT method to known smooth solutions. Smooth analytic solutions to the Euler equations are difficult to find although some do exist. One example is that of the Prandtl-Meyer fan for flow around a smoothly expanding channel. As a more general approach to constructing exact solutions, we 
use the method of analytic solutions, sometimes known as the method of manufactured solutions, whereby one picks an arbitrary smooth solution and includes a forcing term in (1) such that the solution to the forced set of equations is the chosen smooth solution. This approach is very general and we have found it to be an invaluable tool in verifying the implementation of a given numerical approach.

To construct such a solution we take $\mathbf{u}_{s}\left(x_{1}, x_{2}, t\right)$ as a known smooth function. Equation (1) is then modified to

$$
\frac{\partial}{\partial t} \mathbf{u}+\frac{\partial}{\partial x_{1}} \mathbf{f}_{1}(\mathbf{u})+\frac{\partial}{\partial x_{2}} \mathbf{f}_{2}(\mathbf{u})=\mathbf{h}\left(\mathbf{u}_{s}\right),
$$

where $\mathbf{u}, \mathbf{f}_{1}, \mathbf{f}_{2}$ are defined as before and

$$
\mathbf{h}\left(\mathbf{u}_{s}\right)=\frac{\partial}{\partial t} \mathbf{u}_{s}+\frac{\partial}{\partial x_{1}} \mathbf{f}_{1}\left(\mathbf{u}_{s}\right)+\frac{\partial}{\partial x_{2}} \mathbf{f}_{2}\left(\mathbf{u}_{s}\right) .
$$

The boundary conditions are also modified in a similar way. Clearly one solution to the modified problem (23) is $\mathbf{u}=\mathbf{u}_{s}$. The particular choice of $\mathbf{u}_{s}$ is quite arbitrary and for the purposes of this work we use trigonometric functions in both space and time. The choice made here is

$$
\left.\begin{array}{l}
\rho_{s}=\frac{1}{8} \cos \left(\frac{\pi\left(x_{1}-5\right)}{10}\right) \cos \left(\frac{\pi x_{2}}{10}\right) \cos \left(\frac{\pi t}{10}\right)+1 \\
u_{1, s}=\cos \left(\frac{\pi x_{1}}{10}\right) \cos \left(\frac{\pi x_{2}}{10}\right) \cos \left(\frac{\pi t}{10}\right) \\
u_{2, s}=\frac{1}{2} \cos \left(\frac{\pi\left(x_{1}-5\right)}{10}\right) \cos \left(\frac{\pi\left(x_{2}-5\right)}{10}\right) \cos \left(\frac{\pi t}{10}\right) \\
p_{s}=\rho_{s}\left[\frac{1}{4} \cos \left(\frac{\pi x_{1}}{10}\right) \cos \left(\frac{\pi\left(x_{2}-5\right)}{10}\right) \cos \left(\frac{\pi t}{10}\right)+1\right]
\end{array}\right\}
$$

where the conserved quantities are constructed from these given primitives. We consider the solution to (23) on two different domains, the first being a simple square with $\left|x_{k}\right| \leq 2, k=1,2$. For this domain (and for later examples) Cartesian grids are defined by

$$
\begin{gathered}
\mathscr{R}\left(\left[x_{1, a}, x_{1, b}\right] \times\left[x_{2, a}, x_{2, b}\right], N_{1}, N_{2}\right)=\left\{\left(x_{1, a}+i_{1} \Delta x_{1}, x_{2, a}+i_{2} \Delta x_{2}\right) \mid\right. \\
\left.\Delta x_{k}=\left(x_{k, b}-x_{k, a}\right) / N_{k}, i_{k}=0,1, \ldots, N_{k}, k=1,2\right\} .
\end{gathered}
$$

For this example we use $\mathscr{R}([-2,2] \times[-2,2], 40 m, 40 m)$, where $m$ is an integer indicating grid size. The initial condition is taken to be $\mathbf{u}_{s}\left(x_{1}, x_{2}, 0\right)$ and the boundary conditions on the perimeter of the square are given by the exact solution for all time. The modified equations (23) are integrated numerically for $0 \leq t \leq 1$ and the solution error is computed at the final time. Of course if the $\alpha$ 's in Equation (8) are not taken to be 1 the method will not be second order accurate everywhere in the domain. Still it is of substantial interest to determine the actual accuracy of the method for smooth flows such as this. Table 1 shows the maximum error in the primitive 
variables at $t=1$ for various grid resolutions determined by $m$. The convergence rate is computed from one resolution to the next as $\kappa=\log _{2}\left(e_{\rho}(m) / e_{\rho}(2 m)\right)$ as well as a least squares fit of the rates over the entire refinement process which we label $\tilde{\kappa}$. The max-norm convergence rates are generally reasonably close to secondorder. Actual second order convergence is not expected because the method defaults to first order near characteristic extrema as is typical of most limited schemes.

\begin{tabular}{|c|c|c|c|c|c|c|c|c|}
\hline \hline $\mathrm{m}$ & $e_{\rho}(m) \mathrm{F}$ & $\kappa$ & $e_{u_{1}}(m) \mathrm{F}$ & $\kappa$ & $e_{u_{2}}(m) \mathrm{F}$ & $\kappa$ & $e_{p}(m) \mathrm{F}$ & $\kappa$ \\
\hline 1 & $1.1 \mathrm{e}-3$ & - & $4.7 \mathrm{e}-3$ & - & $6.8 \mathrm{e}-4$ & - & $8.6 \mathrm{e}-4$ & - \\
\hline 2 & $7.9 \mathrm{e}-4$ & .48 & $1.3 \mathrm{e}-3$ & 1.9 & $3.9 \mathrm{e}-4$ & .80 & $2.7 \mathrm{e}-4$ & 1.7 \\
\hline 3 & $1.5 \mathrm{e}-4$ & 2.4 & $3.1 \mathrm{e}-4$ & 2.1 & $7.3 \mathrm{e}-5$ & 2.4 & $6.4 \mathrm{e}-5$ & 2.1 \\
\hline 8 & $4.7 \mathrm{e}-5$ & 1.7 & $9.4 \mathrm{e}-5$ & 1.7 & $1.9 \mathrm{e}-5$ & 1.9 & $1.8 \mathrm{e}-5$ & 1.8 \\
\hline \hline$\tilde{\kappa}$ & 1.5 & & 1.9 & & 1.7 & & 1.9 & \\
\hline \hline
\end{tabular}

Table 1 Convergence results for the square domain using the FCT method (indicated by F in table headers. Maximum errors in density, velocity components and pressure at $t=1$ for grid resolutions determined by $m$, and the estimated convergence rates $\kappa=\log _{2}\left(e_{\rho}(m) / e_{\rho}(2 m)\right)$ as well as a least squares fits of the convergence rates over the entire refinement process $\tilde{\kappa}$ are shown.

The second domain considered is a circular disk of radius 0.8 which is discretized using an overlapping grid consisting of a background Cartesian grid given by $\mathscr{R}([-0.6,0.6] \times[-0.6,0.6], 30 \mathrm{~m}, 30 \mathrm{~m})$, and a boundary fitted annular grid defined by $\mathscr{A}((0.0,0.0),[0.4,0.8], 10 \mathrm{~m}, 80 \mathrm{~m})$ with

$$
\begin{aligned}
\mathscr{A}\left(\left(x_{1, c}, x_{2, c}\right),\left[r_{a}, r_{b}\right], N_{r}, N_{\theta}\right)=\left\{\left(x_{1, c}, x_{2, c}\right)+r_{i_{r}}\left(\cos \left(\theta_{i_{\theta}}\right), \sin \left(\theta_{i_{\theta}}\right)\right) \mid\right. \\
\left.r_{i_{r}}=r_{a}+i_{r}\left(r_{b}-r_{a}\right) / N_{r}, \theta_{i_{\theta}}=2 \pi i_{\theta} / N_{\theta}, i_{k}=0,1, \ldots, N_{k}, k=r, \theta\right\} .
\end{aligned}
$$

Through the use of such an overlapping mesh we provide a further check of both the implementation of the scheme on curvilinear grids as well as for the interpolation scheme at grid overlaps. Table 2 shows the maximum error in the primitive variables at $t=1$ for various resolutions of the overlapping grid. We note the near secondorder convergence for each of the variables.

\begin{tabular}{|c|c|c|c|c|c|c|c|c|}
\hline \hline $\mathrm{m}$ & $e_{\rho}(m) \mathrm{F}$ & $\kappa$ & $e_{u_{1}}(m) \mathrm{F}$ & $\kappa$ & $e_{u_{2}}(m) \mathrm{F}$ & $\kappa$ & $e_{p}(m) \mathrm{F}$ & $\kappa$ \\
\hline 1 & $8.6 \mathrm{e}-5$ & - & $2.3 \mathrm{e}-4$ & - & $7.5 \mathrm{e}-5$ & - & $1.1 \mathrm{e}-4$ & - \\
\hline 2 & $2.8 \mathrm{e}-5$ & 1.6 & $6.8 \mathrm{e}-5$ & 1.8 & $2.3 \mathrm{e}-5$ & 1.7 & $3.1 \mathrm{e}-5$ & 1.8 \\
\hline 3 & $7.2 \mathrm{e}-6$ & 2.0 & $2.0 \mathrm{e}-5$ & 1.8 & $6.2 \mathrm{e}-6$ & 1.9 & $7.7 \mathrm{e}-6$ & 2.0 \\
\hline 8 & $2.0 \mathrm{e}-6$ & 1.8 & $6.1 \mathrm{e}-6$ & 1.7 & $1.7 \mathrm{e}-6$ & 1.9 & $2.0 \mathrm{e}-6$ & 1.9 \\
\hline \hline$\tilde{\kappa}$ & 1.8 & & 1.7 & & 1.8 & & 1.9 & \\
\hline \hline
\end{tabular}

Table 2 Convergence results for the circular domain. Maximum errors in density, velocity components, and pressure at $t=1$ for grid resolutions determined by $m$, and the estimated convergence rates $\kappa=\log _{2}\left(e_{\rho}(m) / e_{\rho}(2 m)\right)$ as well as a least squares fits of the convergence rates over the entire refinement process $\tilde{\kappa}$ are shown. 


\subsection{Isolated Contact and Shock Discontinuities}

\subsubsection{Contact wave}

The contact wave is a traveling discontinuous jump where characteristics run parallel to the front. As such, error can accumulate with the result that a nominally $P^{\text {th }}$ order shock capturing scheme will generally converge at the rate of $\kappa=P /(P+1)$ in the $L_{1}$ sense $[28,26,4]$. There are some so-called compressively limited schemes which can achieve $\kappa=1$ convergence although such schemes often have other undesirable characteristics such as the artificial steepening of smooth solutions [41, 55]. The construction of the FCT method does not immediately indicate what the convergence rate should be.

The initial conditions for the contact wave consists of the left state $\left(\rho, u_{1}, u_{2}, p\right)_{L}=$ $(0.1,1.0,0.0,1.0)$ and the right state $\left(\rho, u_{1}, u_{2}, p\right)_{R}=(1.0,1.0,0.0,1.0)$ with the jump at $x_{0}=0.25$. We can construct a weak solution corresponding to a vanishing viscosity solution, and we will call such solutions "exact" with the understanding that there may be many weak solutions. The exact solution to this problem consists of a propagating discontinuity moving to the right with speed 1.0. The density jumps through this discontinuity but the pressure and velocity remain constant. Simulations are performed on the grid defined by $\mathscr{L}([0.0,1.0], 200 m)$ where $m$ is a measure of grid resolution (see equation(20)). A value of $\gamma=1.4$, corresponding to a diatomic ideal gas, is chosen.

A convergence study is performed at various numerical resolutions indicated by $m$ with the comparisons taking place at $t_{f}=0.5$ using the discrete $L_{1}$ norm. Results from this study are given in Table 3. Here it is seen that both the FCT and Godunov

\begin{tabular}{||c||c|c|c|c||}
\hline \hline$m$ & $e_{\rho}(m) \mathrm{F}$ & $\kappa$ & $e_{\rho}(m) \mathrm{G}$ & $\kappa$ \\
\hline \hline 1 & $1.06 \mathrm{e}-2$ & - & $1.39 \mathrm{e}-2$ & - \\
\hline 2 & $6.64 \mathrm{e}-3$ & .67 & $8.78 \mathrm{e}-3$ & .66 \\
\hline 4 & $4.18 \mathrm{e}-3$ & .67 & $5.55 \mathrm{e}-3$ & .66 \\
\hline 8 & $2.63 \mathrm{e}-3$ & .67 & $3.51 \mathrm{e}-3$ & .66 \\
\hline \hline$\tilde{\kappa}$ & .67 & & .66 & \\
\hline \hline
\end{tabular}

Table 3 Convergence results for the contact wave problem using second order Godunov and FCT approximations, indicated by "F" and "G" in the headings respectively. $L_{1}$ errors in density at $t_{f}=0.5$ are computed for grid resolutions determined by $m$. Estimated convergence rates $\kappa=\log _{2}\left(e_{\rho}(m) / e_{\rho}(2 m)\right)$ as well as a least squares fit of the convergence rates over the entire refinement process $\tilde{\kappa}$ are shown. Note that errors for velocity and pressure are identically zero.

methods attain the expected convergence rate of $\approx 2 / 3$ as measured by both $\kappa$ and $\tilde{\kappa}$. We can also see that the FCT method captures the contact with slightly less error than the Godunov method although the results for the Godunov method are sensitive to the choice of Riemann solver and limiter [4]. 


\subsubsection{Shock wave}

Consider a Mach 2 shock with $\gamma=1.4$. The pre- and post-shock states are given by $\left(\rho, u_{1}, u_{2}, p\right)_{L}=(2.67,1.48,0.0,4.5)$ and $\left(\rho, u_{1}, u_{2}, p\right)_{R}=(1.0,0.0,0.0,1.0)$. For this nonlinear phenomenon, the characteristic curves enter into the discontinuity which acts as a natural steepening mechanism. Computations are carried out on the unit interval $x \in[0,1]$ using mesh $\mathscr{L}([0.0,1.0], 200 m)$ with $m$ being a measure of grid resolution. The initial jump is placed at $x_{0}=0.25$ and integration is carried out to $t_{f}=0.25$ where $L_{1}$ errors are computed. The results are presented in Table 4 .

\begin{tabular}{||c||c|c|c|c||c|c|c|c|c|c|c|c||}
\hline \hline$m$ & $e_{\rho}(m) \mathrm{F}$ & $\kappa$ & $e_{\rho}(m) \mathrm{G}$ & $\kappa$ & $e_{u_{1}}(m) \mathrm{F}$ & $\kappa$ & $e_{u_{1}}(m) \mathrm{G}$ & $\kappa$ & $e_{p}(m) \mathrm{F}$ & $\kappa$ & $e_{p}(m) \mathrm{G}$ & $\kappa$ \\
\hline \hline 1 & $8.38 \mathrm{e}-3$ & - & $7.08 \mathrm{e}-3$ & - & $5.59 \mathrm{e}-3$ & - & $4.83 \mathrm{e}-3$ & - & $1.44 \mathrm{e}-2$ & - & $1.26 \mathrm{e}-2$ & - \\
\hline 2 & $3.94 \mathrm{e}-3$ & 1.0 & $3.65 \mathrm{e}-3$ & .96 & $2.91 \mathrm{e}-3$ & .94 & $2.76 \mathrm{e}-3$ & .81 & $6.57 \mathrm{e}-3$ & 1.1 & $6.35 \mathrm{e}-3$ & .99 \\
\hline 4 & $2.08 \mathrm{e}-3$ & .92 & $1.82 \mathrm{e}-3$ & 1.0 & $1.39 \mathrm{e}-3$ & 1.1 & $1.22 \mathrm{e}-3$ & 1.2 & $3.63 \mathrm{e}-3$ & .86 & $3.27 \mathrm{e}-3$ & .96 \\
\hline 8 & $9.63 \mathrm{e}-4$ & 1.1 & $9.15 \mathrm{e}-4$ & .99 & $7.13 \mathrm{e}-4$ & .96 & $6.72 \mathrm{e}-4$ & .86 & $1.66 \mathrm{e}-3$ & 1.1 & $1.60 \mathrm{e}-3$ & 1.0 \\
\hline \hline$\tilde{\kappa}$ & 1.03 & & .99 & & 1.00 & & .97 & & 1.02 & & .99 & \\
\hline \hline
\end{tabular}

Table 4 Convergence results for the shock wave problem using second order Godunov $(\mathrm{G})$ and FCT (F) approximations. $L_{1}$ errors in density, velocity and pressure are shown at $t_{f}=0.25$ for grid resolutions determined by $m$. Estimated convergence rates $\kappa=\log _{2}\left(e_{\rho}(m) / e_{\rho}(2 m)\right)$ as well as a least squares fits of the convergence rates over the entire refinement process $\tilde{\kappa}$ are also shown.

Both schemes have similar $L_{1}$ errors and demonstrate the expected first order convergence with $\kappa \approx 1$ and $\tilde{\kappa} \approx 1$ for density, velocity and pressure. This implies that the number of cells for which there is $O(1)$ point-wise error is fixed which implies that the shock does not continually smear as a function of time. Contrast this to the case of the contact in Section 4.2.1 where the captured discontinuity contains an increasing number of grid cells even as its overall width decreased.

\subsection{Sod's shock tube problem (modified)}

For this example problem we investigate the behavior of the FCT and Godunov methods for a modified version of Sod's shock tube problem. This problem is designed to highlight the poor behavior of some numerical methods near sonic points in rarefaction waves and was previously discussed in Section 3.3 where the sonic fix for the FCT method was described. A description of sonic fixes for Godunov schemes can be found, for example, in [55]. The computational domain is again chosen to be $x \in[-1,1]$, the initial jump is placed at $x_{0}=-0.4$, and the governing equations (1) are integrated to $t_{f}=0.5$. The computational grid for this study is given by $\mathscr{L}([-1.0,1.0], 100 m)$.

The exact density and pressure, as well as approximate results for $m=1$ for both the Godunov and FCT methods, are shown in Figure 3 which demonstrates the sim- 

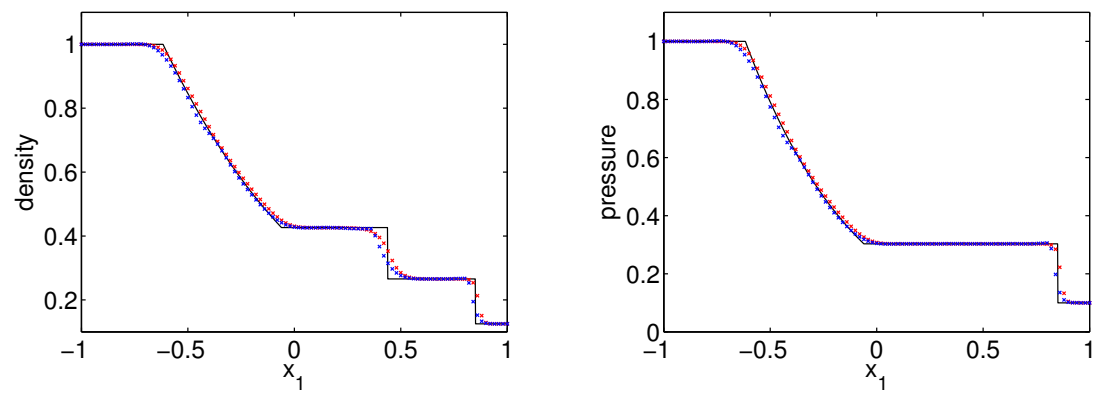

Fig. 3 Exact solution (black line) and numerical approximations with $m=1$ for Godunov's method using Roe's approximate Riemann solver (red marks) and the FCT method (blue marks) for the modified Sod shock tube problem at $t_{f}=0.5$. Shown here are the density (left) and the pressure (right).

ilarity of the two approximate solutions. This trend continues for all resolutions but is more easily seen for this coarse simulation where $m=1$. Figure 3 also shows that both methods seem to be handling the sonic rarefaction. Quantitative convergence results are shown in Table 5 using the discrete $L_{1}$ norm. These results indicate that

\begin{tabular}{||c||c|c|c|c||c|c|c|c||c|c|c|c||}
\hline \hline$m$ & $e_{\rho}(m) \mathrm{F}$ & $\kappa$ & $e_{\rho}(m) \mathrm{G}$ & $\kappa$ & $e_{u_{1}}(m) \mathrm{F}$ & $\kappa$ & $e_{u_{1}}(m) \mathrm{G}$ & $\kappa$ & $e_{p}(m) \mathrm{F}$ & $\kappa$ & $e_{p}(m) \mathrm{G}$ & $\kappa$ \\
\hline \hline 2 & $8.86 \mathrm{e}-3$ & - & $9.44 \mathrm{e}-3$ & - & $1.44 \mathrm{e}-2$ & - & $1.44 \mathrm{e}-2$ & - & $6.54 \mathrm{e}-3$ & - & $6.32 \mathrm{e}-3$ & - \\
\hline 4 & $5.00 \mathrm{e}-3$ & .83 & $5.31 \mathrm{e}-3$ & .83 & $6.99 \mathrm{e}-3$ & 1.0 & $7.51 \mathrm{e}-3$ & .94 & $3.21 \mathrm{e}-3$ & 1.0 & $3.22 \mathrm{e}-3$ & .97 \\
\hline 8 & $3.05 \mathrm{e}-3$ & .71 & $3.03 \mathrm{e}-3$ & .81 & $3.32 \mathrm{e}-3$ & 1.1 & $4.08 \mathrm{e}-3$ & .88 & $1.54 \mathrm{e}-3$ & 1.1 & $1.67 \mathrm{e}-3$ & .94 \\
\hline 16 & $1.83 \mathrm{e}-3$ & .74 & $1.80 \mathrm{e}-3$ & .75 & $1.59 \mathrm{e}-3$ & 1.1 & $2.42 \mathrm{e}-3$ & .75 & $7.24 \mathrm{e}-4$ & 1.1 & $9.08 \mathrm{e}-4$ & .88 \\
\hline \hline$\tilde{\kappa}$ & .76 & & .80 & & 1.06 & & .86 & & 1.06 & & .93 & \\
\hline \hline
\end{tabular}

Table 5 Convergence results for the modified Sod shock tube problem. Discrete $L_{1}$ error and associated convergence rates for the Godunov $(\mathrm{G})$ and FCT (F) schemes at selected resolutions associated with the choice of $m$. Apparently the mesh is of insufficient resolution for the methods to exhibit global convergence rates of $2 / 3$ for the $L_{1}$ norm of density which is dictated by the captured contact.

although both schemes are clearly converging to the exact solution, neither scheme is yet in the asymptotic range of convergence where the $L_{1}$ error of density will be dominated by the $2 / 3$ convergence rate near the contact. Even so, both schemes provide similar convergence behavior with the FCT yielding slightly higher convergence rates for the pressure and velocity. 


\subsection{A Two-Shock Riemann Problem}

The last Riemann problem investigated in this work is commonly known as the twoshock problem. The exact solution to this problem for $\gamma=1.4$ has a $M \approx 5.62$ shock in the rightmost characteristic field, a $M \approx 1.81$ shock in the leftmost characteristic field, and a contact wave separating the two. Left and right states are taken from [55] and given as $\left(\rho, u_{1}, u_{2}, p\right)_{L}=(5.99242,19.5975,0.0,460.894)$ and $\left(\rho, u_{1}, u_{2}, p\right)_{R}=$ $(5.99242,-6.19633,0.0,46.0950)$ The exact solution is determined as in [57], and results in a nearly stationary shock for the leftmost characteristic field. The actual speed of the left shock is $S \approx 0.78$, the velocity through the contact wave is $u_{1} \approx 8.69$, and the rightmost shock moves with speed $S \approx 12.25$. The capturing of the nearly stationary shock proves to be one of the primary difficulties for this problem (see $[38,1]$ for details on slowly moving shocks). Shock capturing schemes also have difficulty representing the contact in this problem and there is a need to accurately resolve that jump before a reasonable global approximation is achieved.

The solution for this problem is approximated for $x \in[-1,1]$ using the mesh $\mathscr{L}([-1.0,1.0], 100 m)$ and integration is carried out to a final time of $t_{f}=0.035$. Figure 3 shows profiles of density and pressure for the exact solution at that time
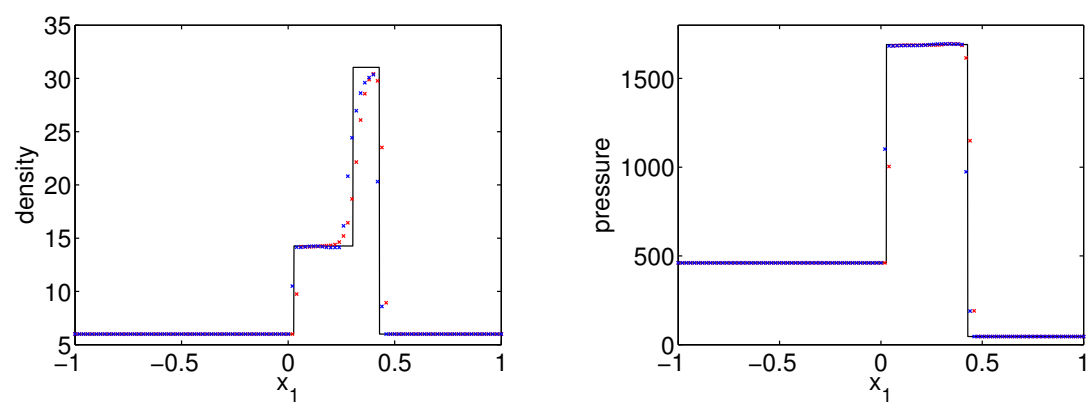

Fig. 4 Exact solution (black line) and approximations with $m=1$ for Godunov's method using Roe approximate Riemann solver (red marks) and the FCT method (blue marks) for the two-shock Riemann problem at $t_{f}=0.035$. Shown here are the density (left) and the pressure (left).

as well as the numerical approximations for $m=1$. Qualitatively it is seen that the two schemes produce similar results, however, close inspection revels the Godunov approximation to be slightly less oscillatory particularly in the pressure while the FCT approximation shows a sharper capture of the contact wave. Table 6 shows quantitative convergence results for the two schemes using the discrete $L_{1}$ norm for the computation of the errors. This table shows that the Godunov approximations demonstrate somewhat higher convergence rates for all quantities, but that for the resolutions discussed here the FCT approximations always give smaller actual errors. In fact for the pressure and velocity, the errors in the FCT approximations are 


\begin{tabular}{||c||c|c|c|c|c|c|c|c||c|c|c|c||}
\hline \hline$m$ & $e_{\rho}(m) \mathrm{F}$ & $\kappa$ & $e_{\rho}(m) \mathrm{G}$ & $\kappa$ & $e_{u_{1}}(m) \mathrm{F}$ & $\kappa$ & $e_{u_{1}}(m) \mathrm{G}$ & $\kappa$ & $e_{p}(m) \mathrm{F}$ & $\kappa$ & $e_{p}(m) \mathrm{G}$ & $\kappa$ \\
\hline \hline 2 & $4.48 \mathrm{e}-1$ & - & $7.53 \mathrm{e}-1$ & - & $1.10 \mathrm{e}-1$ & - & $3.40 \mathrm{e}-1$ & - & $8.74 \mathrm{e} 0$ & - & $3.29 \mathrm{e} 1$ & - \\
\hline 4 & $2.58 \mathrm{e}-1$ & .80 & $4.08 \mathrm{e}-1$ & .88 & $7.15 \mathrm{e}-2$ & .62 & $1.41 \mathrm{e}-1$ & 1.3 & $6.49 \mathrm{e} 0$ & .43 & $1.40 \mathrm{e} 1$ & 1.23 \\
\hline 8 & $1.51 \mathrm{e}-1$ & .77 & $2.26 \mathrm{e}-1$ & .85 & $2.48 \mathrm{e}-2$ & 1.5 & $7.78 \mathrm{e}-2$ & .86 & $3.34 \mathrm{e} 0$ & .96 & $7.89 \mathrm{e} 0$ & .83 \\
\hline 16 & $9.00 \mathrm{e}-2$ & .75 & $1.43 \mathrm{e}-1$ & .66 & $1.66 \mathrm{e}-2$ & .58 & $4.76 \mathrm{e}-2$ & .71 & $1.82 \mathrm{e} 0$ & .88 & $4.94 \mathrm{e} 0$ & .66 \\
\hline \hline$\tilde{\kappa}$ & .77 & & .80 & & .91 & & .94 & & .75 & & .90 & \\
\hline \hline
\end{tabular}

Table 6 Discrete $L_{1}$ error and associated convergence rates for the two shock problem using the Godunov $(\mathrm{G})$ and FCT (F) schemes at selected resolutions associated with the choice of $m$. Neither scheme is yet in the asymptotic range of convergence where the $L_{1}$ errors in density will be dominated by the $2 / 3$ convergence rate at the contact.

more than three times smaller than the Godunov approximations at coarse resolutions and still more than twice as small for the finest mesh considered.

\subsection{Shu-Osher Problem}

The final one-dimensional test case considered in this chapter is a problem originally considered by Shu and Osher [36] and subsequently by others [24, 48]. This problem consists of a $M=3$ shock in air, $\gamma=1.4$, traveling into unshocked air with sinusoidally perturbed density. As originally presented, the problem has a number of parameters and the specific values used here are taken from [24]. The initial setup is

$$
\begin{array}{lll}
\rho=3.857143, & u_{1}=2.629369, & u_{2}=0, p=10.33333 \\
\rho=1-\varepsilon \sin (\lambda \pi x), & u_{1}=0, & \text { for } x_{1}<-4 \\
u_{2}=0, p=1 & \text { for } x_{1} \geq-4
\end{array}
$$

where the parameter values are $\varepsilon=0.2$ and $\lambda=5$. The approximate solution is computed for $x \in[-5,5]$ using $\mathscr{L}([-5.0,5.0], 200 \mathrm{~m})$ and integrated to a final time $t_{f}=1.8$.

When interpreting results, it is useful to understand the Riemann structure of the solution when $\varepsilon=0$. For this case we can determine an exact solution and the waves present there give a good indication where structures in the more complicated solution will arise. When $\varepsilon=0$, the solution consists of a $M=3$ shock traveling with speed $S \approx 3.55$. The perturbed problem, $\varepsilon \neq 0$ and small, will have disturbances traveling along the other two characteristic fields with speeds $S \approx 2.63$ and $S \approx 0.69$. At $t=1.8$, the lead shock will have travelled to $x_{1} \approx 2.39$, the contact wave to $x_{1} \approx 0.73$ and the left acoustic wave to $x_{1} \approx-2.76$. For small $\varepsilon$ it is expected that the exact solution will change character near these locations.

A reference solution, computed with $m=128$ up to $t=1.8$, can be seen, for example, in Figure 5. For $x<-2.76$ the solution is the unperturbed post-shock state. For $x \in(-2.76,0.73)$ the solution exhibits mild oscillations in all quantities. These oscillations are the result of the passage of the left acoustic wave. For $x \in$ 
$(0.73,2.39)$ the solution exhibits high frequency oscillations. Notice that for the computational resolution $m$, the high frequency oscillations in the density for $x \in$ $(0.73,2.39)$ contain approximately $2 m$ grid points per wavelength. The solution with $m=128$ uses a sufficiently fine grid to resolve these oscillations as evidenced by the fact that further refinement does not change the character of the solution, and because it results in approximately 256 cells per wavelength for $x \in(0.73,2.39)$. For $x>2.39$ the solution returns to the initial upstream state. The locations where the solution changes behavior are, as expected, those mentioned above in the discussion of the Riemann structure for $\varepsilon=0$.

There is no known closed form solution to this problem and convergence results must be estimated through comparison to more finely resolved solutions. Here we use a method similar to that presented in [33]. At a given point, $x_{i}$, we assume the solution at a given resolution differs from the exact solution by

$$
\mathbf{u}_{e}\left(x_{i}\right)-\mathbf{u}_{m}\left(x_{i}\right) \approx \mathbf{c}\left(x_{i}\right) h_{m}^{\kappa}
$$

where $\mathbf{u}_{e}$ is the exact solution, $\mathbf{u}_{m}$ the numerical approximation, $\mathbf{c}\left(x_{i}\right)$ depends only on $x_{i}, \kappa$ is the convergence rate and $h_{m}$ is the grid spacing. Note that we have uniform grid spacing. From (26) one can compute

$$
\left\|\mathbf{u}_{m_{1}}(x)-\mathbf{u}_{m_{2}}(x)\right\|_{h} \approx \| \mathbf{c}(x)||_{h}\left|h_{m_{1}}^{\kappa}-h_{m_{2}}^{\kappa}\right|
$$

using a discrete norm. Numerical approximations at three resolutions and equation (27) can be combined to produce two equations which define the convergence rate $\kappa$ and the constant $\|\mathbf{c}(x)\|_{h}$. The solution error can then be approximated as $e_{\mathbf{u}}(m)=\left\|\mathbf{u}_{e}-\mathbf{u}_{m}\right\|_{h} \approx\|\mathbf{c}(x)\|_{h} h^{\kappa}$. When estimating the error and convergence rate for a given approximation with resolution given by $m$, we use the three approximations $\mathbf{u}_{m}, \mathbf{u}_{64}$ and $\mathbf{u}_{128}$. Table 7 shows the convergence results using the discrete $L_{1}$

\begin{tabular}{|c|c|c|c|c|c|c|c|c|c|c|c|c|}
\hline$m$ & $e_{\rho}(m) \mathrm{F}$ & $\kappa$ & $e_{\rho}(m) \mathrm{G}$ & $\kappa$ & $e_{u_{1}}(m) \mathrm{F}$ & $\kappa$ & $e_{u_{1}}(m) \mathrm{G}$ & $\kappa$ & $e_{p}(m) \mathrm{F}$ & $\kappa$ & $e_{p}(m) \mathrm{G}$ & $\kappa$ \\
\hline 1 & $1.16 \mathrm{e} 0$ & .75 & $1.20 \mathrm{e} 0$ & 44 & $3.44 \mathrm{e}-1$ & 1.1 & $3.02 \mathrm{e}-1$ & .94 & $2.34 \mathrm{e} 0$ & 1.1 & $1.98 \mathrm{e} 0$ & \\
\hline 2 & $9.18 \mathrm{e}-1$ & 86 & $1.01 \mathrm{e} 0$ & 52 & $1.57 \mathrm{e}-1$ & 1.1 & $1.55 \mathrm{e}-1$ & .94 & $1.08 \mathrm{e} 0$ & 1.1 & $1.08 \mathrm{e} 0$ & $\overline{93}$ \\
\hline 4 & $7.86 \mathrm{e}-1$ & 1.1 & $8.64 \mathrm{e}-1$ & .67 & $6.35 e-2$ & 1.1 & $7.85 e-2$ & $\overline{92}$ & $4.75 \mathrm{e}-1$ & 1.1 & $5.67 \mathrm{e}-1$ & 94 \\
\hline 8 & $5.98 \mathrm{e}-1$ & 1.4 & $7.28 \mathrm{e}-1$ & .93 & $3.10 \mathrm{e}-2$ & 1.1 & $4.22 \mathrm{e}-2$ & .94 & $2.23 \mathrm{e}-1$ & 1.1 & $2.94 \mathrm{e}-1$ & 93 \\
\hline 16 & $2.39 \mathrm{e}-1$ & 1.4 & $5.00 \mathrm{e}-1$ & 1.3 & $1.52 \mathrm{e}-2$ & 1.2 & $2.35 \mathrm{e}-2$ & 1.0 & $1.06 \mathrm{e}-1$ & 1.2 & $1.70 \mathrm{e}-1$ & 1.1 \\
\hline 32 & $8.90 \mathrm{e}-2$ & 1.4 & $2.19 \mathrm{e}-1$ & 1.5 & $6.87 \mathrm{e}-3$ & 1.4 & $1.20 \mathrm{e}-2$ & 1.3 & $4.57 \mathrm{e}-2$ & 1.3 & $8.38 \mathrm{e}-2$ & 1.3 \\
\hline
\end{tabular}
norm for both the FCT and Godunov schemes. From this table it is clear that the

Table 7 Convergence results for the Shu-Osher test problem using both the Godunov (G) and FCT (F) methods. Convergence rates and errors are computed with (26) and (27) using finely resolved simulations at $m=64$ and $m=128$.

coarser resolutions do not approximate the solution well at all, particularly for the density, and low rates of convergence are attained. Figures 5 and 6 demonstrate this graphically where the numerical approximations for $m=1$ are plotted on top of the 
reference solution. Figure 5 shows the global character of the solution and Figure 6 shows a zoom of the density in the most oscillatory region. For low resolutions,
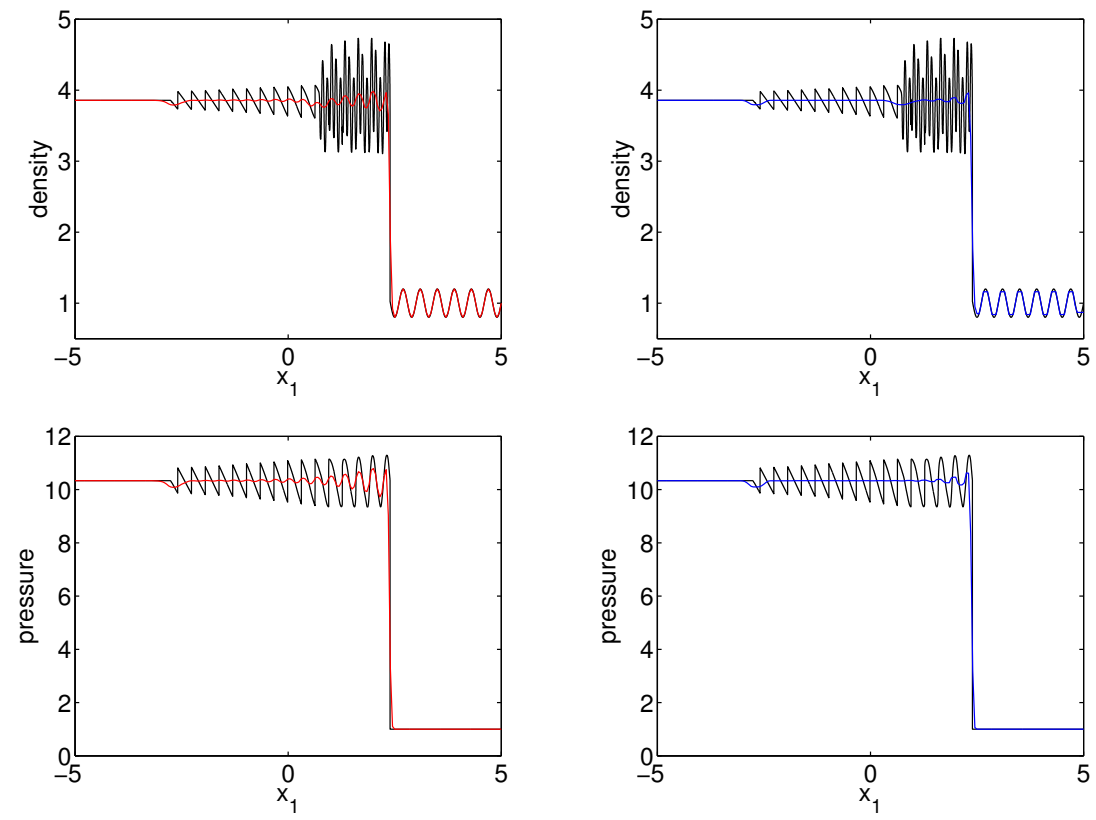

Fig. 5 Comparison of the numerical approximations with $m=1$ at $t=1.8$ for the Shu-Osher test problem. For all images the black line represents the reference solution with $m=128$ while the red line (left) shows the Godunov approximation and the blue line (right) shows the FCT approximation. From top to bottom are density and pressure.

the high frequency oscillations are not well represented and both methods exhibit poor convergence properties, particularly for the density as seen in Figure 6. This is reflected by the convergence rates which are less than 1 . At some critical resolution however, both methods see a rise in convergence rates, tending to some value larger than 1. Once this transition occurs, the high frequency oscillations begin to be well represented as shown in Figures 7 and 8. This transition to higher convergence rates happens at lower resolution for FCT, indicating that it has more resolving power than the Godunov method. For the highest resolutions demonstrated here, both approximations are reasonably representing all structures in the flow and their convergence rates become roughly equal. However, because the FCT method experienced the transition to higher convergence rates earlier in the refinement process, the errors at the highest resolutions are smaller than for the Godunov approximations by nearly a factor of 2 . 


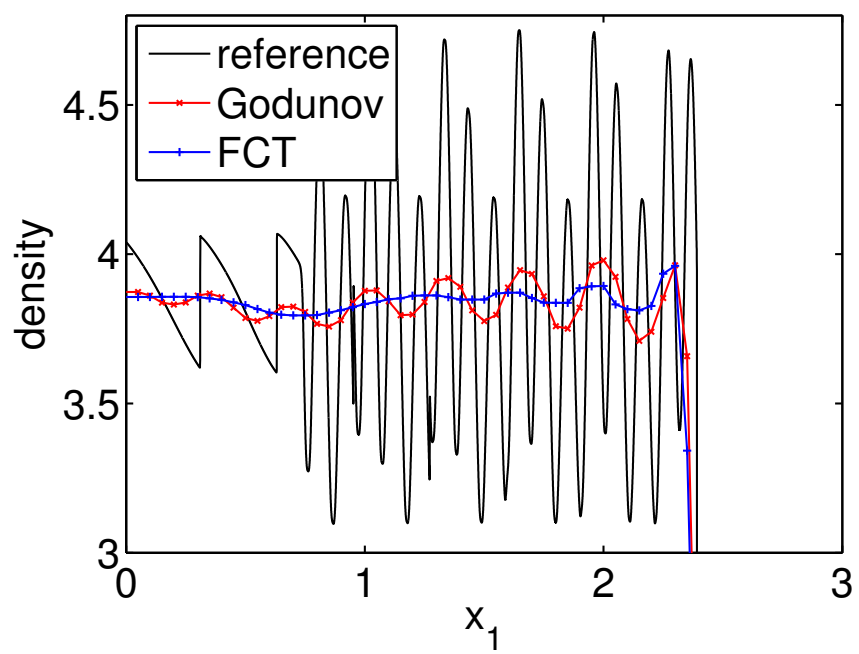

Fig. 6 Zoom of density near the high frequency oscillations. Shown are the FCT and Godunov approximations wtih $m=1$, and the reference solution computed by the Godunov method with $m=128$.

\subsection{Shock Impingement on Stationary Cylinder}

The first two-dimensional test problem which we consider is the impingement of a $M=2$ shock on a rigid immovable cylinder. The basic problem consists of a rigid cylinder of radius 0.5 placed in the larger domain $[-2,2] \times[-2,2]$. A Mach 2 shock initially located at $x_{1}=-1.5$ runs from left to right. The computational mesh is defined as the overlapping grid constructed from an annulus $\mathscr{A}((0,0),[0.5,1.0], 10 \mathrm{~m}, 80 \mathrm{~m})$ and a rectangle $\mathscr{R}([-2,2] \times[-2,2], 80 \mathrm{~m}, 80 \mathrm{~m})$, where $\mathscr{A}$ and $\mathscr{R}$ are defined as before in Section 4.1. The boundary around the cylinder is defined as a slip wall (see [32]), the left boundary as an inflow, and the remaining boundaries are given outflow conditions. Phenomena of interest are limited to those associated with the shock/cylinder interaction. Provided that the simulation is not run too far in time, waves generated at the cylinder do not reach the exterior boundaries and so the exterior boundary condition choice has little influence. Figure 9 shows the computational mesh as well as color contours of density for the initial conditions. Numerical values for the initial conditions in primitive quantities, corresponding to a Mach 2 shock in air $(\gamma=1.4)$, were shown previously in Section 4.2.2.

The comparisons carried out in this chapter use the resolution $m=1$ displayed in Figure 9 for the coarse grid simulation. Adaptive mesh refinement (AMR) is then used for successive resolutions. For this test of shock interaction with a single cylinder, additional levels of AMR use a factor four refinement in each coordinate 

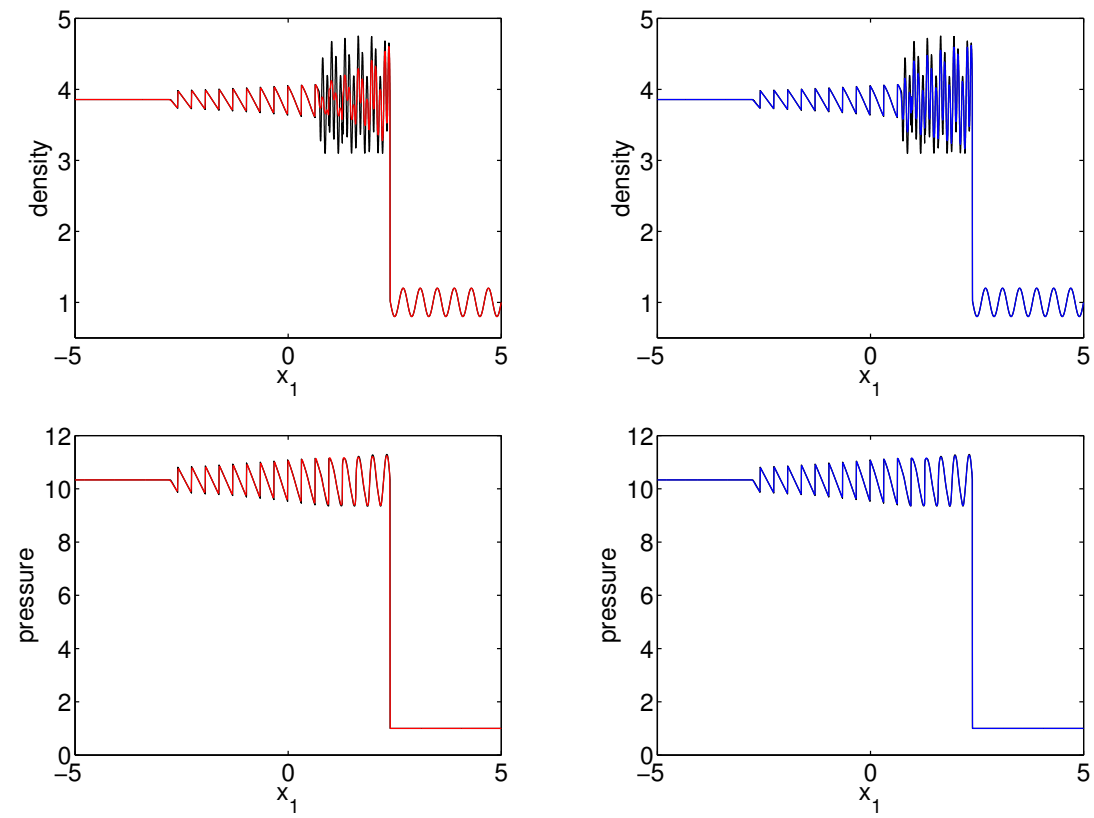

Fig. 7 Comparison of the numerical approximations at $t=1.8$ for the Shu-Osher test problem and $m=16$. For all images the black line represents the reference solution with $m=128$ while the red line (left) shows the Godunov approximation and the blue line (right) shows the FCT approximation. From top to bottom are density and pressure.

direction and so the four resolutions investigated have approximate grid spacings $h \approx 0.05,0.0125,0.003125$, and 0.00078125 . Notice that because the initial condition uses a perfect jump, there exists numerical artifacts along the $c^{-}$characteristic and contact path. No effort is made to remove these and their contribution may be seen throughout the simulations.

Figure 10 shows the computed density using both methods for $t=0.6, t=1.0$, and $t=1.4$ as the incident shock reflects from the cylinder boundary. Overall the results show remarkably good agreement although slight differences can be seen at $t=1.4$ in the low density wake region of the cylinder. To give a better indication of what is happening, Figure 11 shows the AMR grid structure, numerical Schlieren images [5], and the estimated error in density at $t=1.4$. The computation of the error estimate will be discussed below. The image of the AMR grids is perhaps the most informative because it demonstrates the increased noise created by the FCT method. Numerical noise tends to flag cells for refinement by the AMR algorithm and so a larger portion of the domain is covered by fine meshes for the FCT simulation. This type of noise, also interpretable as staircases [39], is a common phenomenon in FCT simulations. There are ways to reduce the noise, such as adding higher levels of artificial diffusion, using different high order fluxes, and others, but in our experience, 


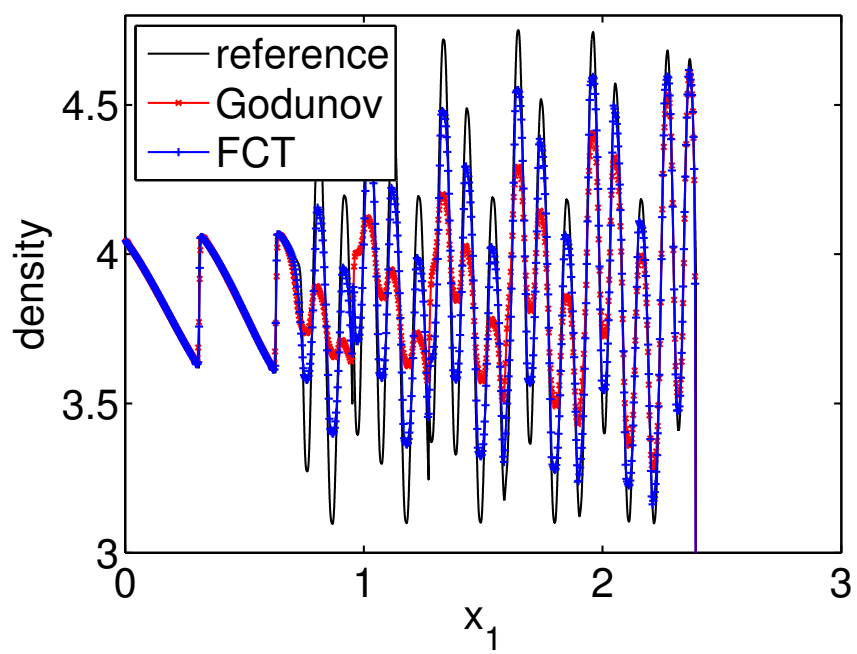

Fig. 8 Zoom of density near the high frequency oscillations. Shown are the FCT and Godunov approximations wtih $m=16$, and the reference solution computed by the Godunov method with $m=128$.
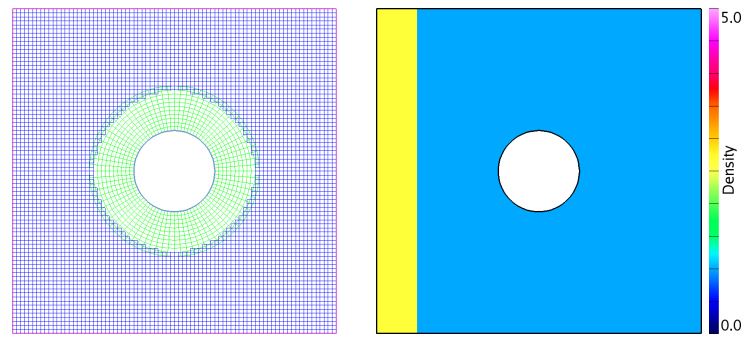

Fig. 9 Overlapping grid structure (left) and color contour of the initial density (right). The overlapping grid structure is used to capture geometry and additional adaptive grids will be dynamically added to locally increase resolution. Note that we only require one layer of interpolation points at grid overlap as discussed in Section 3.1. The initial density shows a $M=2.0$ shock in air (ideal gas with $\gamma=1.4$ ) moving from left to right.

there is no single method which completely eliminates it. On the other hand the results from the Godunov method show little sign of this phenomenon and the AMR meshes conform closely to the locations of rapid change, such as shocks and contacts. The plots of estimated error also show increased noise in the FCT solution. It is worth noting that the remnant of the initial condition on the $c^{-}$characteristic has flagged refinement for the FCT solution whereas this feature has been smoothed by the Godunov method. 

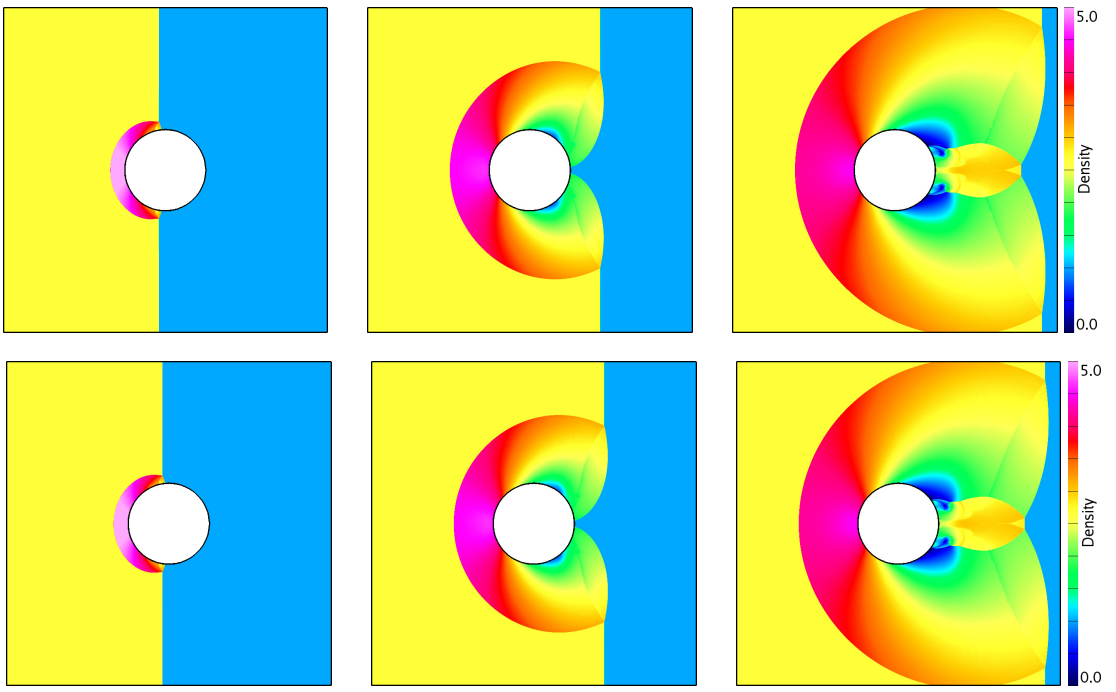

Fig. 10 Color contours of density for the finest resolution using FCT (top) and Godunov's method (bottom) at $t=0.6$ (left), $t=1.0$ (middle), and $t=1.4$ (right).
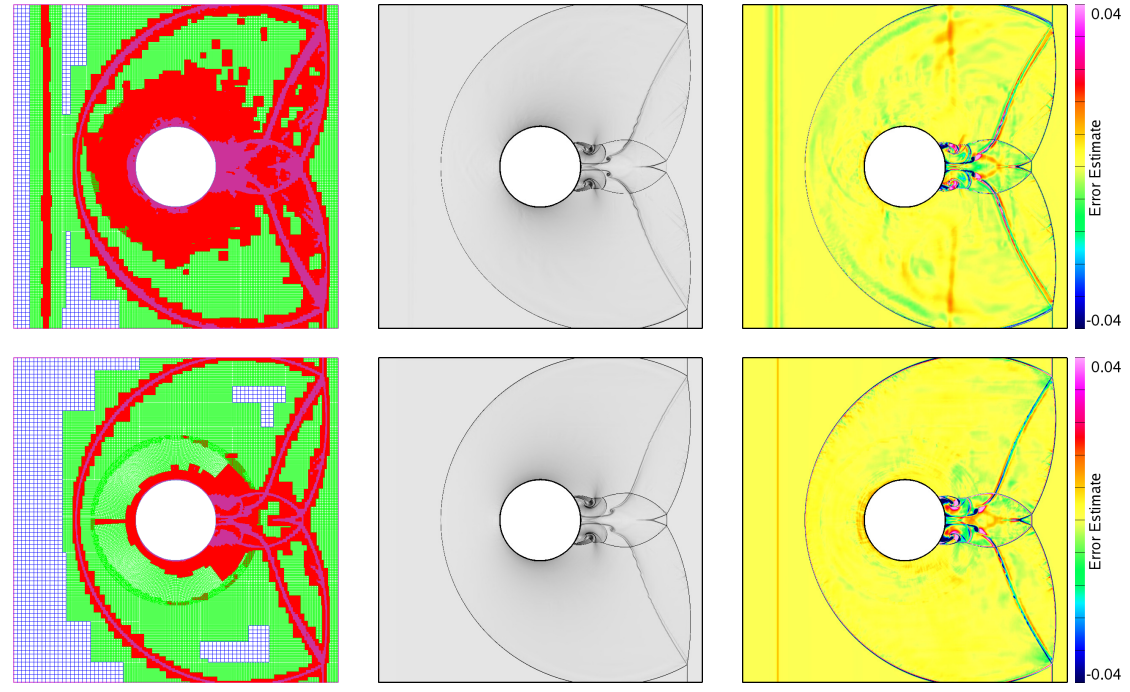

Fig. 11 AMR grid structure (left), numerical Schlieren images (center) and estimated $L_{1}$-error in density (right) for the FCT method (top) and Godunov's method (bottom) for the finest resolution simulation at $t=1.4$ 
Figure 12 shows line plots of the approximations along the lines $x_{2}=0$ with $x_{1} \in[0.5,2.0]$ and $x_{1}=1$ with $x_{2} \in[0,2]$ which gives an indication of convergence as the mesh is refined. From these plots one can again see the trend that FCT approx-
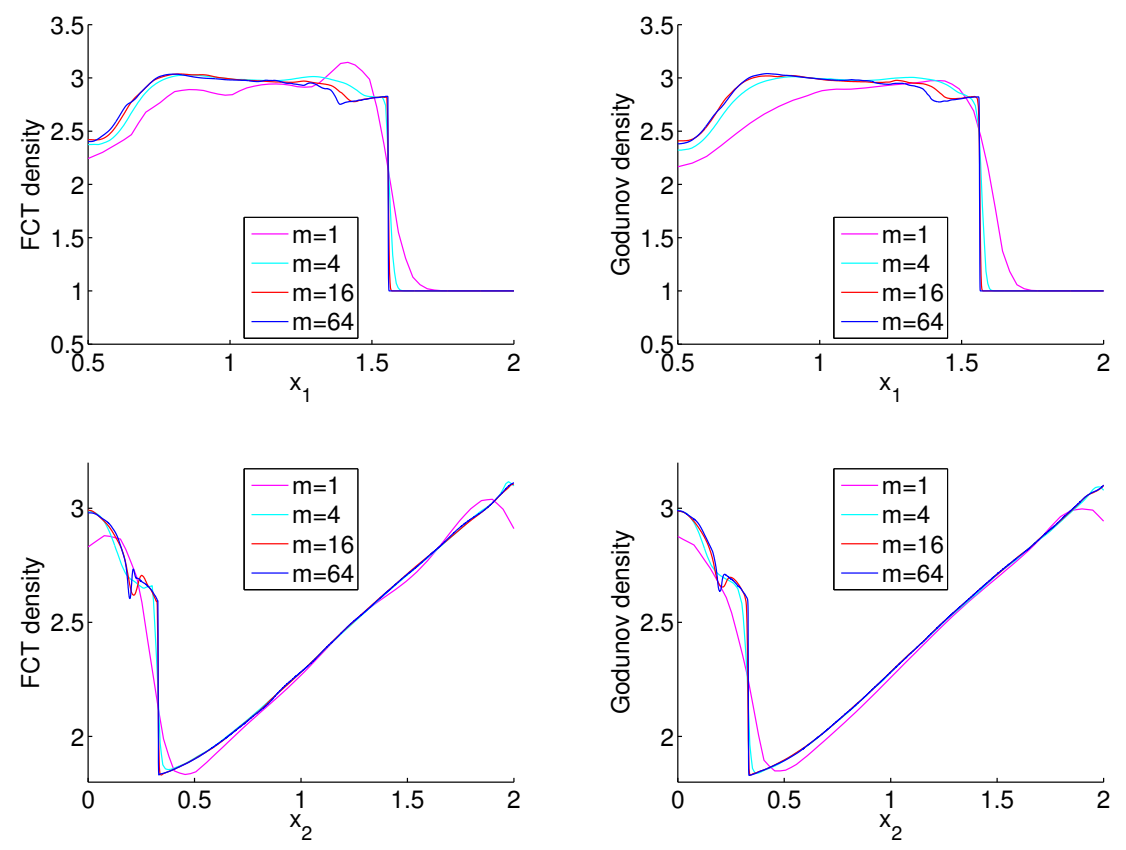

Fig. 12 Solution convergence along the lines $x_{2}=0$ (top) and $x_{1}=1$ (bottom) for the FCT method (left) and the Godunov method (right) on the shock-cylinder problem. Shown here is the density with the colors indicating numerical resolution. Maroon represents the solution with no AMR while cyan, red, and finally blue represent 1,2 , and 3 levels of additional factor four adaptive meshes respectively.

imations contain more noise as compared to the Godunov approximations which generally vary more smoothly. Table 8 shows estimated $L_{1}$-norm self convergence errors and convergence rates. These errors and rates were computed using the finest three resolutions following the approach presented in [33] and as outlined in Section 4.5. An advantage to this method is that it naturally provides an estimate for the exact solution $\mathbf{u}_{e}$ in equation (26). This result can be used to estimate solution errors as was done to obtain the error estimates shown in Figure 11. The results in Table 8 show that the errors and convergence rates are similar for the FCT and Godunov methods. It is interesting to see that convergence results for all quantities, including the velocities and pressure which do not jump through contact waves, show sublinear convergence. The probable cause for this behavior is the complex interactions of shocks, contacts and rarefactions as well as the instabilities in the wake region of 


\begin{tabular}{||c||c|c||c|c||c|c||c|c||}
\hline \hline$m$ & $e_{\rho}(m) \mathrm{F}$ & $e_{\rho}(m) \mathrm{G}$ & $e_{u_{1}}(m) \mathrm{F}$ & $e_{u_{1}}(m) \mathrm{G}$ & $e_{u_{2}}(m) \mathrm{F}$ & $e_{u_{2}}(m) \mathrm{G}$ & $e_{p}(m) \mathrm{F}$ & $e_{p}(m) \mathrm{G}$ \\
\hline \hline 4 & $1.54 \mathrm{e}-2$ & $1.91 \mathrm{e}-2$ & $1.02 \mathrm{e}-2$ & $1.32 \mathrm{e}-2$ & $7.55 \mathrm{e}-3$ & $8.77 \mathrm{e}-3$ & $3.00 \mathrm{e}-2$ & $3.75 \mathrm{e}-2$ \\
\hline 16 & $5.60 \mathrm{e}-3$ & $7.49 \mathrm{e}-3$ & $3.70 \mathrm{e}-3$ & $5.76 \mathrm{e}-3$ & $3.13 \mathrm{e}-3$ & $3.00 \mathrm{e}-3$ & $1.06 \mathrm{e}-2$ & $1.45 \mathrm{e}-2$ \\
\hline 64 & $2.03 \mathrm{e}-3$ & $2.94 \mathrm{e}-3$ & $1.35 \mathrm{e}-3$ & $2.52 \mathrm{e}-3$ & $1.29 \mathrm{e}-3$ & $1.03 \mathrm{e}-3$ & $3.73 \mathrm{e}-3$ & $5.60 \mathrm{e}-3$ \\
\hline \hline$\kappa$ & 0.73 & 0.67 & 0.73 & 0.60 & 0.64 & 0.77 & 0.75 & 0.69 \\
\hline \hline
\end{tabular}

Table 8 Computed errors and convergence rates for the FCT $(\mathrm{F})$ and Godunov $(\mathrm{G})$ schemes on the problem of shock-cylinder interaction. Here we use a weighted $L_{1}$-norm.

the flow. Indications of this are given by the error estimates of Figure 11 where the wake region is shown to have large errors over a substantial area.

\subsection{Irregular Mach Reflection of a Strong Shock}

The next test considered in this chapter is that of irregular Mach reflection of a strong shock at an inclined ramp. This classic example has been investigated by many authors $[60,58,48]$ as well as demonstrated experimentally [21]. In this problem, a Mach 10 shock impacts a ramp which is inclined $30^{\circ}$ from the normal shock propagation direction. The result is a complex interaction and results in an irregular Mach reflection. Numerically, this flow can cause a carbuncle like instability [47, 20] for some numerical methods if proper care is not taken.

Traditionally this test problem has been solved by inclining the incident shock to a Cartesian grid and using special boundary conditions to simulate the transition region at the start of the ramp. For the simulations presented in this chapter, the geometry of a $30^{\circ}$ ramp is realized using overlapping grids and then a Mach 10 shock is impacted onto this ramp. The overlapping grid we use consists of a thin boundary fitted mesh to model the ramp in union with a background Cartesian mesh for the remaining bulk of the computational domain. The background Cartesian mesh is defined by the rectangle $\mathscr{R}([-0.5,3.0] \times[0,1.7], 420,340)$. Although the boundary fitted mesh is not described by a simple mathematical expression, a verbal description will suffice for our purpopses. The physical boundary of this ramp grid is defined as a curve that smoothly transitions from the line $x_{2}=0$, to the line $x_{2}=x_{1} / \sqrt{3}$, and finally to the line $x_{2}=1.4438$. These transitions are defined in terms of integrals of hyperbolic tangent functions and are therefore smooth [29]. The ramp grid is extruded along normals into the domain and the mesh spacing is chosen to approximately match that of the background Cartesian grid. The resulting overlapping grid is shown in Figure 13 where both the full geometry and a zoom near the ramp initiation at the origin are shown. At the scale of the full geometry it is difficult to see the rounding of the corners, but the close up image makes this rounding clear. A rounded corner will have some effect on the solution as it compares to a solution obtained using a perfectly sharp corner. Such effects have been studied for example 

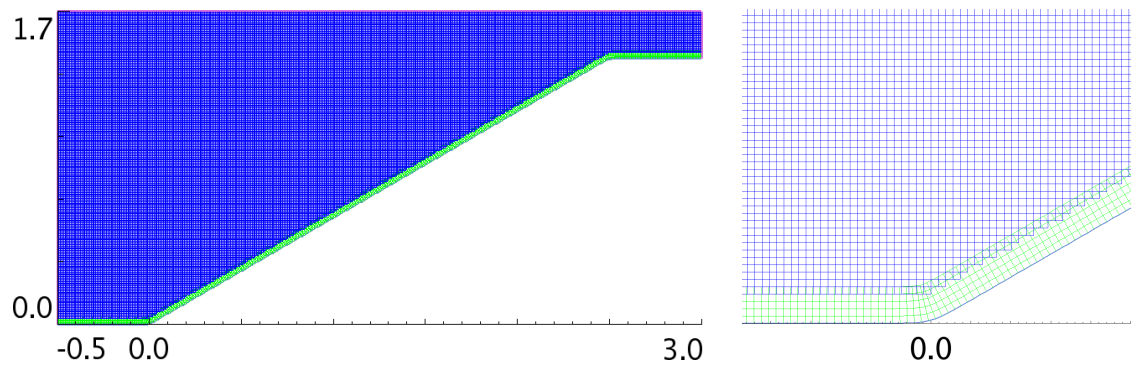

Fig. 13 Basic overlapping grid used for the simulation of irregular Mach reflection on an inclined ramp. The boundary fitted ramp grid is seen in green and the background Cartesian mesh in blue. The full geometry (left) shows what looks like sharp transitions to represent the ramp corners, but the zoom near the origin (right) shows that these corners are very slightly rounded.

in $[37,31]$ and found to be of little consequence when the radius of curvature is small as compared to the flow features of interest (as is the case here).

Initial conditions for a Mach-10 planar shock in air $(\gamma=1.4)$ are $\left(\rho, u_{1}, u_{2}, p\right)_{L}=$ $(8.0,8.25,0.0,116.5)$ and $\left(\rho, u_{1}, u_{2}, p\right)_{R}=(1.4,0.0,0.0,1.0)$. The initial shock is located at $x_{1}=-0.25$ (for reference the left-most boundary is $x_{1}=-0.5$ and the ramp incline begins at $x_{1}=0$ ) and time integration is performed to $t=0.2$. Boundary conditions are set using a slip wall condition along the ramp boundary, inflow conditions along the left boundary and outflow conditions elsewhere. For these simulations, the base mesh has roughly equal mesh spacing throughout the domain with $h \approx \frac{1}{120}$. Simulations are performed at four resolutions starting with only the base mesh and then progressing through to use one additional level of factor four refinement, two additional levels of factor three refinement, and finally two additional levels of factor four refinement.

Figure 14 shows the approximations obtained using the FCT and Godunov methods at the finest resolution with approximate mesh spacing $h \approx 5.21 \times 10^{-4}$. At this scale there are some apparent differences that merit mention. First notice the increased noise production by the FCT method as shown by the increased proportion of the domain flagged for AMR refinement. Also both simulations retain remnants of the initial condition along the $c^{-}$characteristic and contact path. These remnants are covered with fine AMR meshes, although the refinement for the FCT algorithm covers a larger region. Finally it is seen that the minimum pressure inside the main vortex is lower for the FCT simulation than for Godunov.

Figure 15 shows close-up numerical Schlieren images near the main vortex structure at the four different mesh resolutions. For both simulation techniques, the main vortex is poorly represented at low resolutions but with increasing mesh resolution the main features begin to develop. The roll-ups along the slip lines become pronounced for both methods with the Godunov solution showing slightly more detailed structure. The final two solutions show interesting differences in the development of the main vortex. For the Godunov method it remains as a coherent single vortical structure, while for the FCT method it begins to break down and show more 

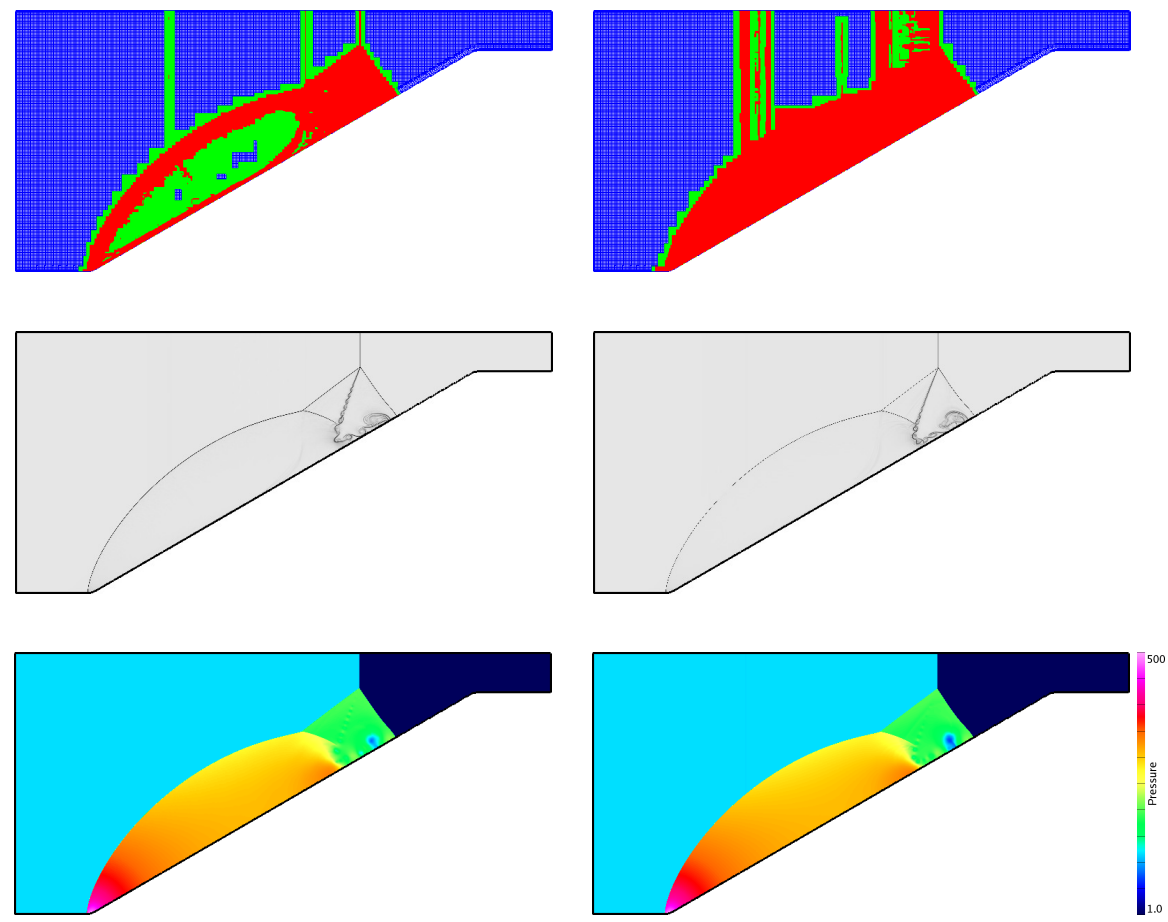

Fig. 14 Simulation results at $t=0.2$ for the Godunov method (left) and FCT (right) with 3 levels of factor 4 refinement. Shown are the AMR mesh (top), a numerical Schlieren image (middle), and pressure (bottom). Notice the increase in noise produced by the FCT method as evidenced by the larger region flagged for AMR refinement. Also notice the lower pressure achieved by the FCT method within the main vortex.

complex behavior. Comparing this behavior with what is seen in [48] shows that the Godunov methods (for [48] the PPM method) tend to maintain a coherent single structure, while the other methods (the hybrid WENO method in [48] and FCT here) produce a vortex which begins to loose coherence at very high resolution. This type of behavior calls into question the limit processes of the various schemes and whether the various methods are in fact approaching the same vanishing viscosity solution. This is an interesting question and will be the subject of future work. As a further comparison of the methods, Figure 16 compares the peak vorticity and minimum scaled temperature, defined as $p / \rho$, for the two methods as a function of grid resolution. For both schemes the minimum temperature decreases and the maximum vorticity increases as the mesh is refined. The FCT results show a lower temperature and smaller vorticity as compared to the Godunov results. A self convergence study is performed as was done in Section 4.6 using a weighted $L_{1}$-norm. The finest three resolutions are used for this comparison and results presented in Table 9. Here it is seen that the performance of the two methods is similar. The $L_{1}$-norm convergence 

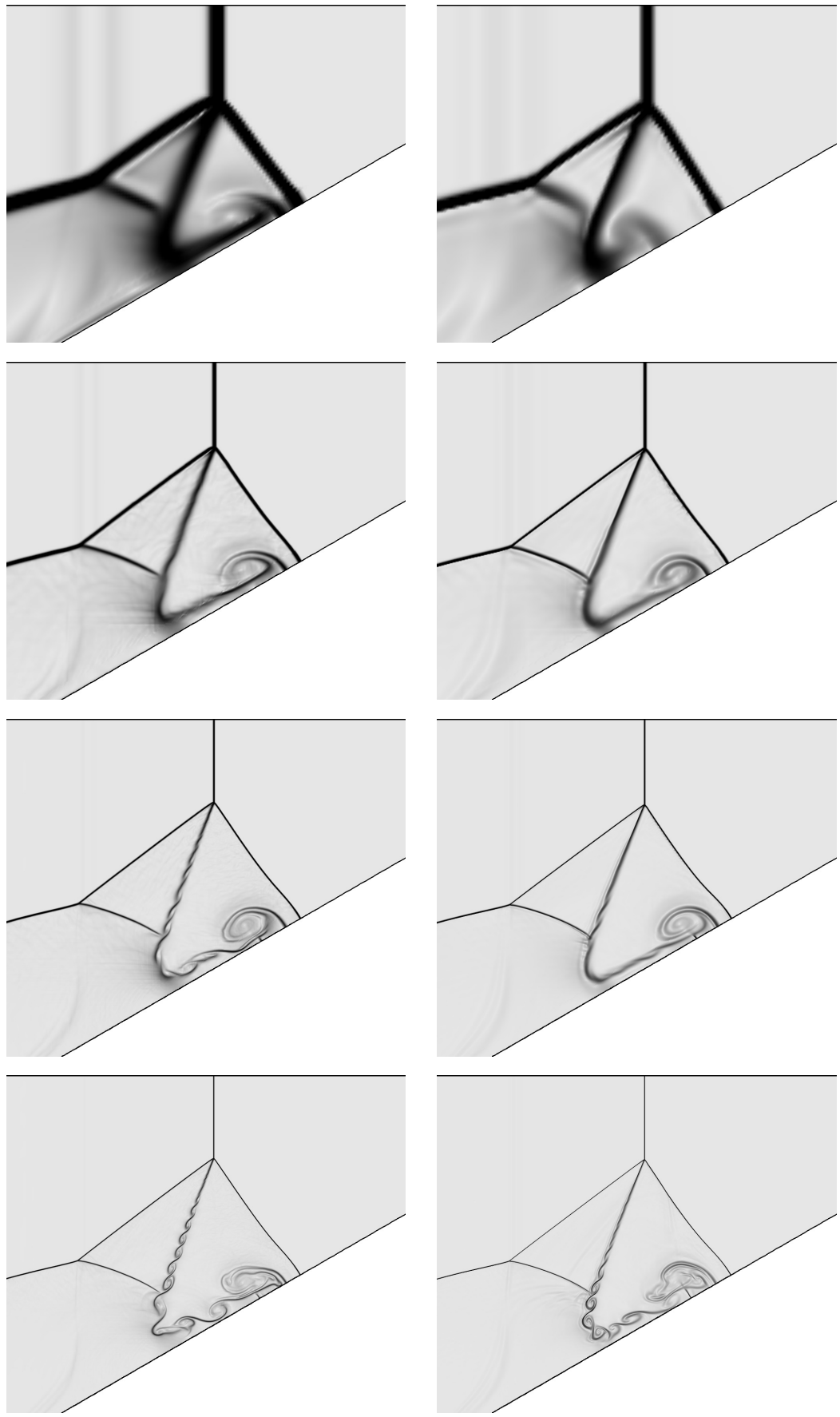

Fig. 15 Zoom of the numerical Schlieren images near the triple point and main vortex for Godunov (left) and FCT (right). Resolution increases from top to bottom with approximate grid spacings $h \approx 1 / 120,1 / 480,1 / 1080$, and $1 / 1920$ respectively. 

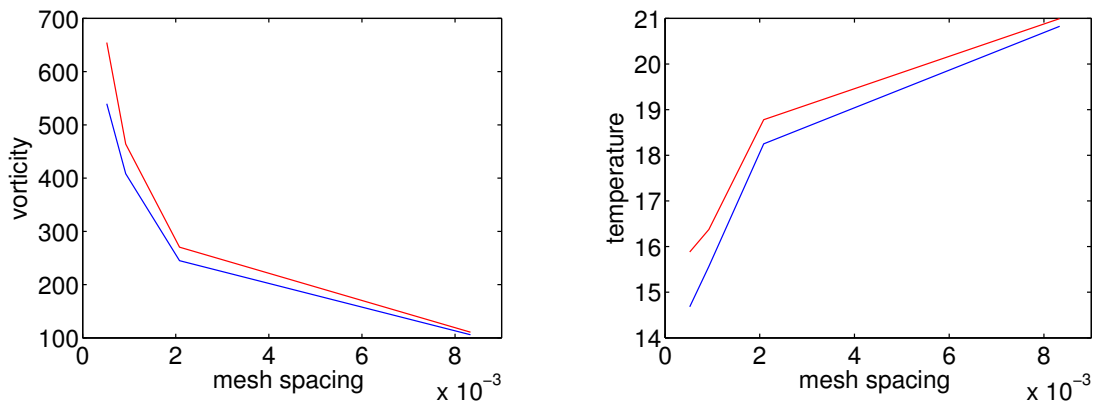

Fig. 16 Maximum vorticity within the main vortex as a function of mesh spacing (left) and minimum scaled temperature (right), defined as $p / \rho$, as a function of mesh spacing. Godunov results are given in red and FCT results in blue.

\begin{tabular}{||c||c|c||c||c||c|c||c|c||}
\hline \hline$m$ & $e_{\rho}(m) \mathrm{F}$ & $e_{\rho}(m) \mathrm{G}$ & $e_{u_{1}}(m) \mathrm{F}$ & $e_{u_{1}}(m) \mathrm{G}$ & $e_{u_{2}}(m) \mathrm{F}$ & $e_{u_{2}}(m) \mathrm{G}$ & $e_{p}(m) \mathrm{F}$ & $e_{p}(m) \mathrm{G}$ \\
\hline \hline 4 & $5.67 \mathrm{e}-2$ & $6.29 \mathrm{e}-2$ & $3.15 \mathrm{e}-2$ & $3.45 \mathrm{e}-2$ & $3.32 \mathrm{e}-2$ & $3.89 \mathrm{e}-2$ & $1.06 \mathrm{e} 0$ & $1.09 \mathrm{e} 0$ \\
\hline 9 & $3.71 \mathrm{e}-2$ & $4.25 \mathrm{e}-2$ & $1.74 \mathrm{e}-2$ & $2.32 \mathrm{e}-2$ & $2.05 \mathrm{e}-2$ & $2.84 \mathrm{e}-2$ & $6.85 \mathrm{e}-1$ & $6.81 \mathrm{e}-1$ \\
\hline 16 & $2.75 \mathrm{e}-2$ & $3.22 \mathrm{e}-2$ & $1.14 \mathrm{e}-2$ & $1.76 \mathrm{e}-1$ & $1.46 \mathrm{e}-2$ & $2.28 \mathrm{e}-2$ & $5.04 \mathrm{e}-1$ & $4.87 \mathrm{e}-1$ \\
\hline \hline$\kappa$ & 0.52 & 0.48 & 0.73 & 0.49 & 0.59 & .39 & 0.54 & 0.58 \\
\hline \hline
\end{tabular}

Table 9 Computed errors and convergence rates for the FCT (F) and Godunov (G) schemes on the problem of irregular Mach reflection. Here we use a weighted $L_{1}$ norm.

rates are somewhat low but this is attributed to the large variations in the solution and unstable vortical flows which arise at the slip line which is evident in Figure 15.

\subsection{Shock impingement on a collection of cylinders}

Section 4.6 presented a problem where a single cylinder was impacted by a Mach 2 shock. Although this problem is by no means trivial it is not unduly complicated either and so we extend this problem to a Mach 2 shock impacting many cylinders. In principal any number of cylinders could be used, but for this case we choose the somewhat arbitrary number of seven. The geometry is meshed using the overlapping grid generated as the union of the seven annuli

$\mathscr{A}((-1,-1),[0.4,0.55], 119 m, 6 m)$,

$\mathscr{A}((-1.2,0),[0.23,0.37], 68 m, 6 m)$,

$\mathscr{A}((-0.75,0.75),[0.3, .45], 89 \mathrm{~m}, 6 \mathrm{~m})$,

$\mathscr{A}((0.2,-1.0),[0.3,0.45], 89 \mathrm{~m}, 6 \mathrm{~m})$,

$\mathscr{A}((0.3,0.6),[0.15,0.3], 44 m, 6 m)$,

$\mathscr{A}((0.7,-0.2),[0.2,0.35], 59 \mathrm{~m}, 6 \mathrm{~m})$,

$\mathscr{A}((-0.05,-0.15),[0.1,0.25], 29 m, 6 m)$, 
and a background grid $\mathscr{R}([-2,4] \times[-2,2], 200 m, 132 m)$ where $m$ is an indicator of grid size. Figure 17 shows the initial mesh corresponding to $m=1$ along with the initial condition as a numerical Schlieren image. As in Section 4.6, the exact
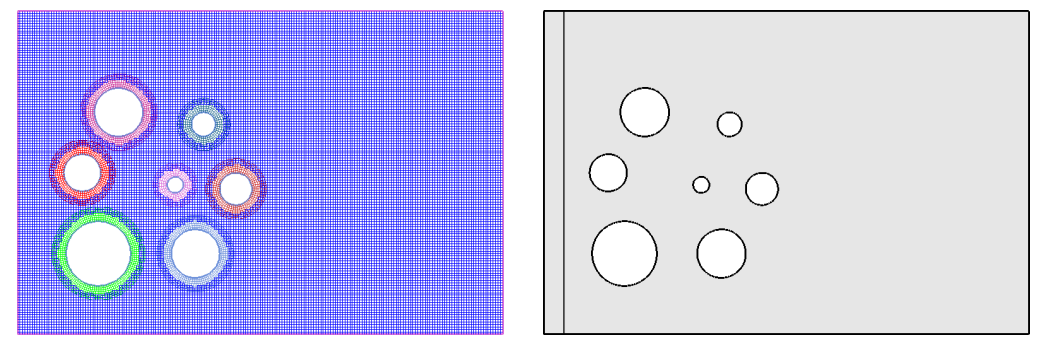

Fig. 17 Computational mesh with resolution corresponding to $m=1$ (left) and initial condition as a numerical Schlieren image(right). The exact shock jump conditions for a Mach 2 shock in air found in Section 4.2.2.

shock jump conditions for a Mach 2 shock in air, as given in Section 4.2.2, are applied as initial conditions at $x_{1}=-1.75$. The boundary conditions are set using an inflow condition at the left, outflow at the top, bottom, and right, and solid slipwall along all cylinder boundaries. The basic idea is to make the boundaries of the square transparent so that the simulation can be viewed as a group of cylinders in a region of very large extent. Section 4.6 provided a good baseline for the interaction of a shock with one cylinder but for this case this basic interaction will be carried out many times and the subsequent interactions will become quite complex.

Figure 18 shows numerical Schlieren images and pressure contours on the grid corresponding to $m=4$ at $t=0.5,1.0,1.5$, and 2.0. Here it is seen that the early time behavior near $t=0.5$ is rather simple and one can easily draw the comparison to the simulations of Section 4.6. As time progresses however, the structure becomes markedly more complex and by $t=2.0$ the simulation contains extremely complicated structures. Still the FCT method is capable of capturing these complexities quite nicely and without undue numerical artifacts. We would also like to note that the collective action of the cylinders is to generally retard the progress of the shock much as the single cylinder did in Section 4.6. This is witnessed by the fact that the lead shock becomes concave forward for both cases at late times indicating that the cylinders were an impedance.

We would also like to give some indication of grid convergence for the solution and so Figure 19 shows simulation results at $t=2.0$ for grid resolutions corresponding to $m=1,2$, and 4 . It is easily seen that even for the lowest resolution the numerical method nicely captures most bulk flow features. Increasing grid resolution serves to sharpen these bulk features as well as to bring out the more detailed fine scale structures.

A final level of complexity is added to this simulation in that the cylinders are now allowed to move. The extension of the FCT algorithm for moving grids is very 

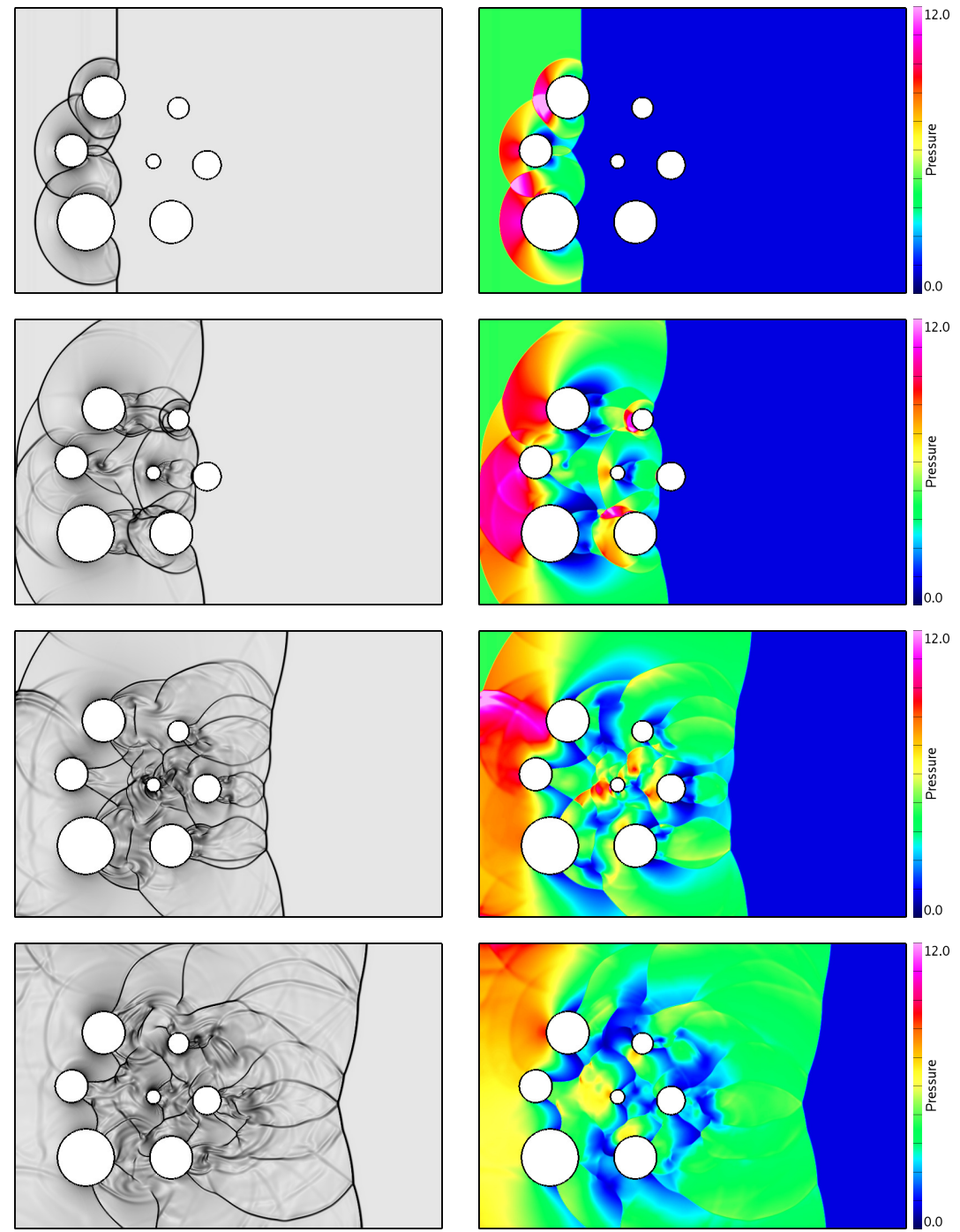

Fig. 18 Numerical Schlieren images (left) and the pressure images (right) of simulations carried out on the grid whose resolution corresponds to $m=4$. The times for these images (from top to bottom) are $t=0.5,1.0,1.5$, and 2.0 . 

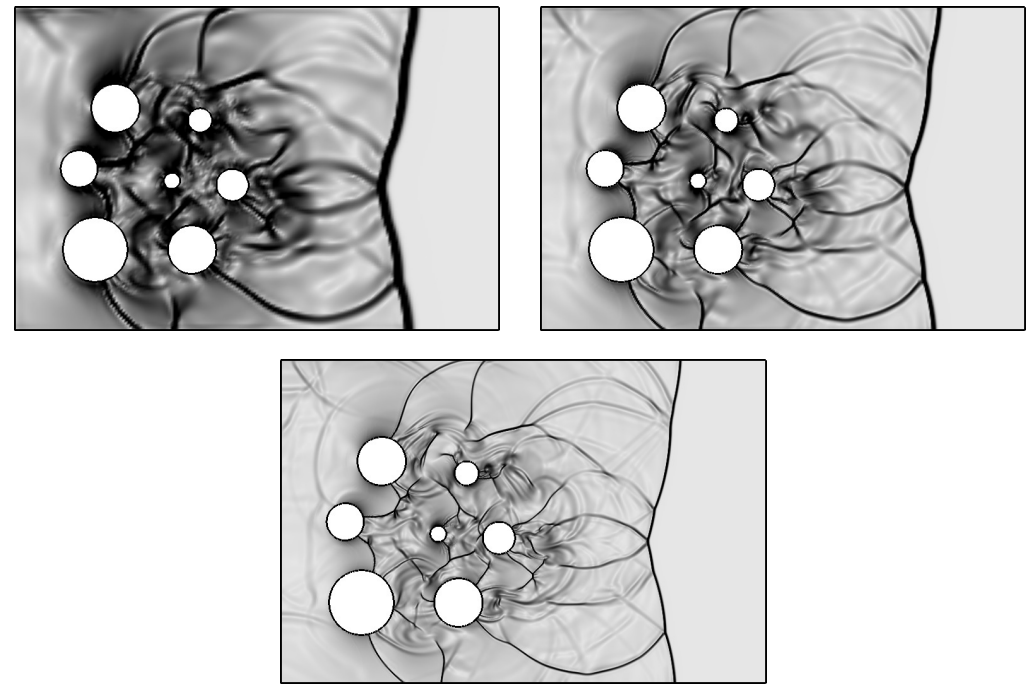

Fig. 19 Numerical Schlieren images at $t=2.0$ for grid resolutions corresponding to $m=1$ (top left), $m=2$ (top right), and $m=4$ (bottom).

straightforward and follows closely the ideas enumerated in [32]. The critical extension from what is presented in Section 3 is simply that the apparent flow velocity on a given component grid is the difference between the grid velocity and the flow velocity. Simulations are then carried out just as before and coupled to the motion of each cylinder through Newton's laws of motion. For this case we assume that all cylinders are made of a material with density 1.5 in dimensionless units and the mass is then computed using this assumption. Figure 20 shows results for the computation on a mesh corresponding to $m=4$ and compares these results to the fixed cylinder case. The FCT algorithm again performs well and maintains sharp interfaces and shocks throughout the simulation without undue numerical artifacts. It is of physical interest to note that while the fixed cylinders retarded the shock wave, the movable cylinders actually aid its progress. This is shown by the generally concave forward shock for the fixed cylinders and the generally convex forward shock for the movable cylinders at late time.

\subsection{An Idealized Z-pinch Implosion}

The last example presented in the FCT evaluation is a complex and challenging prototype of an idealized Z-pinch like magnetic implosion modeled by an extended Euler system with source terms [8]. A schematic diagram of the geometry for an idealized liner implosion is presented in Figure 21. Briefly, in a typical Z-pinch magnetic implosion, a very large total current $I$ (e.g. $20 \mathrm{MA}$ ) with a characteristic rise 

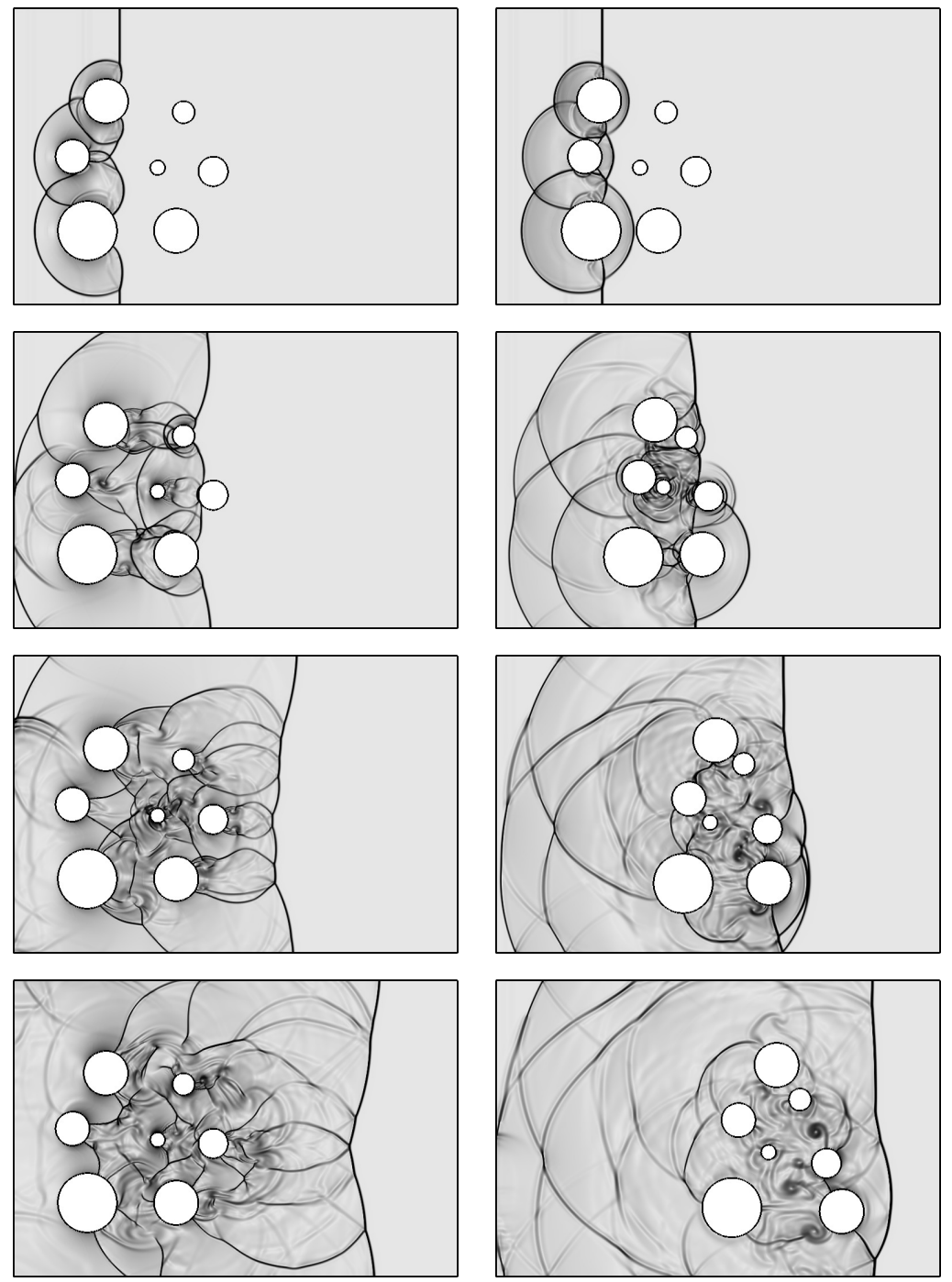

Fig. 20 Numerical Schlieren images of shock interaction with fixed (left) and movable (right) rigid cylinders at $t=0.5$ (top), 1.0 (second row), 1.5 (third row), and 2.0 (bottom). Both simulations were carried out on the mesh corresponding to $m=4$. For the rigid case the cylinders serve to retard the shock resulting in a concave forward shock front while for the movable case the cylinders enhance the shock resulting in a convex forward shock front. 
time of about 100ns is carried initially by either a thin cylindrical metallic liner or a cylindrical array of metallic wires that ablate and produce a plasma [42]. The current flowing through the conductor/plasma produces a corresponding strong azimuthal magnetic flux, $\mathbf{B}_{\theta}$. The induced local plasma current, $\mathbf{J}$ and magnetic flux density, B, produces a strong $\mathbf{J} \times \mathbf{B}$ Lorentz force which accelerates a highly energetic plasma to stagnate on axis in about $100 \mathrm{~ns}$. This stagnation and subsequent magnetic energy conversion produces an intense $10 \mathrm{~ns}$ X-ray radiation pulse [43, 53]. The intense X-ray pulse can then be used for radiation-material interaction studies [44, 42, 45], environments for indirect drive inertial confinement fusion (ICF) applications [42, 45, 43], or for pursuing laboratory-based astrophysics environments $[45,51,54,15,9,22]$. An important limiting mechanism for the amount of radiation energy produced by a Z-pinch is the Magnetic Raleigh-Taylor (MRT) instability which distorts the outer and inner interfaces of the collapsing plasma shell and broadens the pulse width [42, 45, 23].

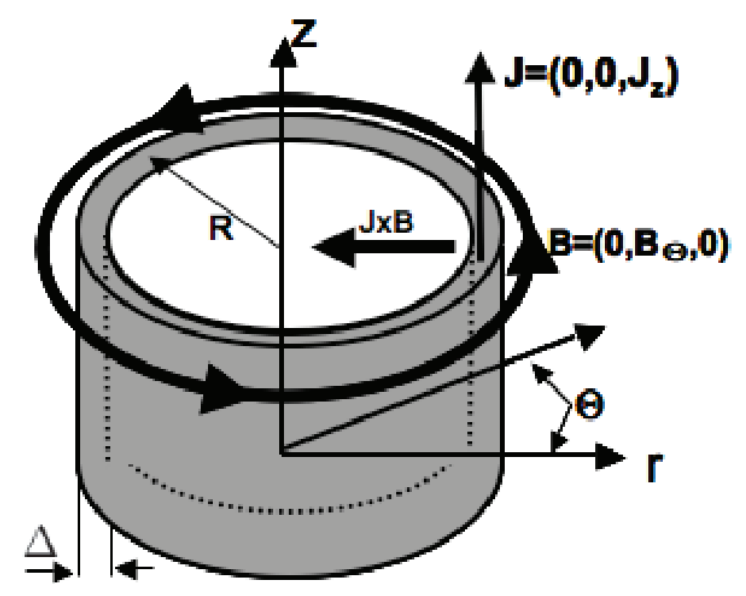

Fig. 21 Schematic of prototype thin-shell implosion model.

The prototype implosions considered in [8], and briefly described in the next subsections, are developed by a simple source term model that can be used in a basic Euler solver for inviscid compressible gases to generate implosions that have the essential character of actual Z-pinch implosions. These problems include simple idealized implosions that can be used as verification type problems, for code development purposes, and also for complex implosions with high material velocities, strong-shocks and compressive heating that may generate a radiation power pulse. 


\subsubsection{An Extended Euler System Model}

A generalization of the Euler conservation law system to a balance equation with general source term is

$$
\frac{\partial \mathbf{u}}{\partial t}+\nabla \bullet \mathbf{f}+\mathbf{s}=\mathbf{0} .
$$

In this equation we have included a general source term $\mathbf{s}$ that will be defined for each individual system of interest. As described in [8] Z-pinch like implosions can be developed by including parameterized source terms for the momentum and energy balance equations that in effect model a Lorentz force and magnetic energy work term along with the definition of an advected scalar that defines the current flow through the liner material. For this model the Euler single temperature system with the advected scalar, $\lambda$, is then defined by

$$
\begin{gathered}
\mathbf{u}=\left[\begin{array}{c}
\rho \\
\rho \mathbf{v} \\
E \\
\rho \lambda
\end{array}\right], \quad \mathbf{f}=\left[\begin{array}{c}
\rho \mathbf{v} \\
\rho \mathbf{v} \otimes \mathbf{v}-\mathbf{T} \\
E \mathbf{v}-\mathbf{T} \cdot \mathbf{v}+\mathbf{q} \\
\mathbf{v} \rho \lambda
\end{array}\right] \\
\mathbf{s}=\left[\begin{array}{c}
0 \\
-\Upsilon \\
-r \cdot \mathbf{v}+Q_{\text {rad }} \\
0
\end{array}\right] .
\end{gathered}
$$

Here the effective Lorentz force source term, $\Upsilon=\mathbf{J} \times \mathbf{B}$, can be evaluated from

$$
\Upsilon=\lambda \rho(I(t))^{2} \frac{1}{r_{\mathrm{eff}}} \hat{\mathbf{e}}_{r} .
$$

In this term, the effective $r$-coordinate, $r_{\text {eff }}$, is used to define the strength of the source term locally. As in [8] the thin-shell Lorentz force term is modified to produce an effective limiting compression ratio by using an effective radial distance, $r_{\text {eff }}$, intended to remove the singularity at $r=0$ with the definition

$$
r_{\text {eff }} / R_{0}=\max \left(r / R_{0}, 1.0^{-4}\right) .
$$

The non-dimensional scalar $0 \leq \lambda(r, t) \leq 1$ is used to control the magnitude and localize the application of the non-dimensional Lorentz force term. Notionally the scalar $\lambda(r, 0)$ is considered to define the initial "current sheet" and to localize the application of the source term during the evolution of the implosion. Thus by specifying $I(t)$ the forcing term magnetically drives the Z-pinch like implosion with the required $1 / r$ behavior in the active region where the Lorentz force term is non-zero. Therefore by including this force term, in a suitably non-dimensionalized form of the Euler equations, a parameterized set of prototype magnetically driven shockhydro problems can be developed. In the examples that follow an analytic limiting case of a thin-shell implosion in the $(x, y)$ plane, Rayleigh-Taylor instability effects 
in a $(r, z)$ implosion and a simple radiating implosion is carried out to evaluate the FCT algorithm.

\subsubsection{Thin-shell Implosion in the $x-y$ Plane and a Comparison with an Asymptotic Analytic Solution}

In this model, the conducting plasma is considered to be concentrated (or lumped) into a asymptotically thin shell with the geometry of Figure 21 and limited to the $(\mathrm{x}, \mathrm{y})$ plane. The non-dimensional initial conditions are choosen with an interior density of $\rho_{\text {pre-fill }}=1.0 \times 10^{-6}$, a liner density of $\rho_{L}=1.0$, and an exterior density of $\rho_{\text {exterior }}=5.0 \times 10^{-7}$ as in [8]. The initial pressure throughout the domain is $p_{0}=1.0 \times 10^{-6}$ and the flow is initially stagnant. In the context of the asymptotic thin-shell implosion analysis presented in [8] this problem uses a current drive of $I(t)=\sqrt{12\left(1-t^{4}\right) t^{2}}$. This current drive results in a power law relation for the radial position $r(t) / R_{0}=1-t^{4}$ for the location of the thin shell as a function of time. In order to give a reasonable intuitive sense for these initial conditions as well as the computational domain where the simulation is carried out refer to Figure 22. The base computational grid which is used for these simulations is shown in Figure 22. Here an annular grid (blue in the figure) is used over much of the domain with the singularity at the origin covered by a square grid (green in the figure). The grid spacing in the radial direction as well as for the center square patch is chosen to be $h=0.025$, and the average grid spacing in the azimuthal direction for the annular grid is $h \approx 0.059$. Solid slip-wall boundary conditions are applied at the left and bottom, and an inflow condition is applied at the outer curved boundary. A series of results for a shell of fixed thickness $\Delta=0.05$ is presented using increasing amounts of AMR. For the initial resolution no AMR is used. The second resolution uses one additional level of AMR with a refinement factor of 4 in each direction giving the effective resolution of $h=0.00625$. The final resolution takes 2 additional levels of AMR each with a refinement factor of $4(h=1 / 640)$. The initial condition for density for the finest resolution simulation is presented in Figure 22. Here the thin ring of conducting material is seen as pink which represents $\rho=1.0$ and $\lambda=1.0$. Interior and exterior to this ring the density is so low $\left(\approx 10^{-6}\right)$ that one cannot distinguish it from a pure vacuum using the included colorbar. Figure 23 shows two snapshots of the density at $t=0.5$, and $t=0.9$. These two images show the imploding conducting shell which appears to remain quite cylindrically symmetric even after its transit from the annular grid to the center square patch. It can be seen, particularly at the later time $t=0.9$, that the thin shell model is clearly approximate. The most obvious indication of this is the fact that the peak density now has a much higher value $\rho_{\max } \approx 1.75$, which results from non-uniformities in the simulation as well as because the shell here is not infinitely thin and compressional effects are induced that are not accounted for in the thin-shell model [8].

For this problem Figure 24 presents the shell radius, $R(t)$, and the shell velocity $V(t)$. The values for these plots are obtained from the simulation results but because we are dealing with simulation results obtained using a capturing code, the selection 

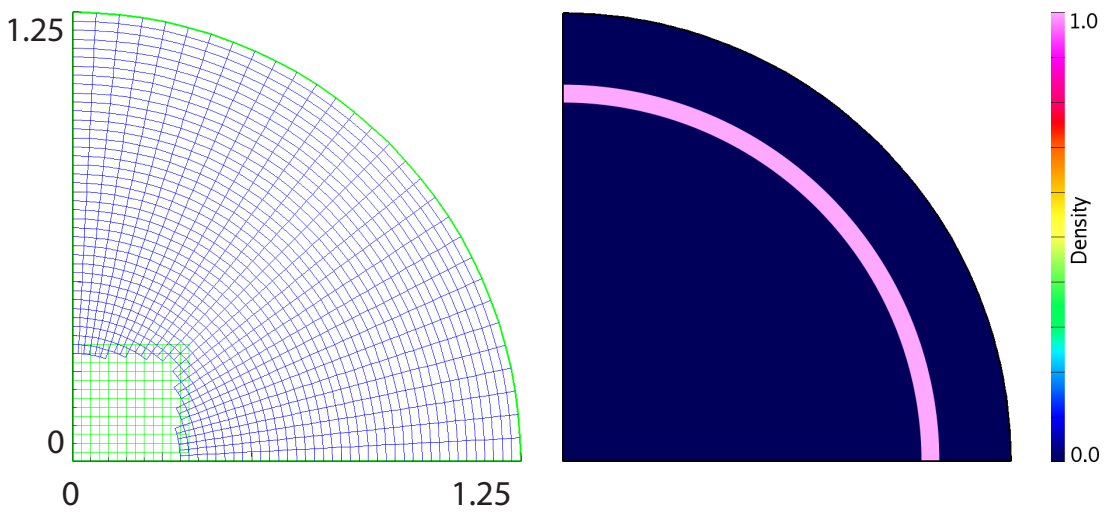

Fig. 22 Left: Computational grid used for the magnetic implosion simulations. Here an annular grid (blue) is used for much of the domain with the singularity covered by a square patch (green) in the center. Right: Initial condition of density for for the highest resolution simulation of the simple implosion. Notice the thin shell of high density surrounded by nearly rarefied regions interior and exterior. The non-dimesnional values for these densities are $\rho_{L}=1.0, \rho_{\text {pre-fill }}=1.0 \times 10^{-6}$ and $\rho_{\text {exterior }}=5.0 \times 10^{-7}$.
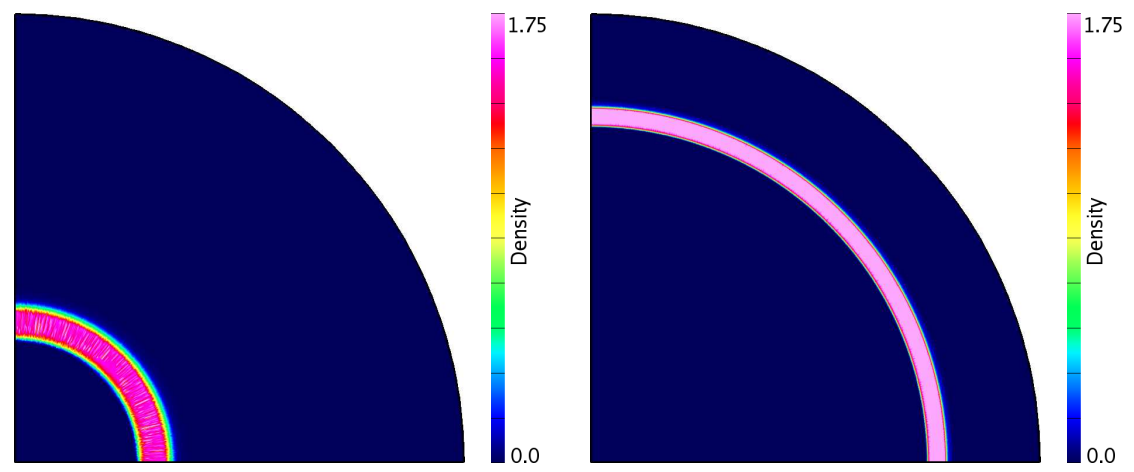

Fig. 23 Density for the highest resolution simulation at $t=0.5$ (right), and $t=0.9$ (left). The thin shell is seen to implode in a remarkably symmetric fashion even after the transition from the annular grid to the square center patch.

of the location, and hence velocity, for the imploding shell is open to interpretation. In these results the shell location at a given time is defined by the location of the computational cell nearest to the origin whose density is $1 / 2$ of the maximum density at that time. From this cell the radius and velocity are selected and used in the plots. In these plots the clustering of the data about the analytic solution demonstrates good agreement of the simulation with the predicted behavior for the thin shell model. One can also see the solution for finer mesh resolutions converging to the analytic solution at all times. This plots show the excellent agreement of the FCT simulation with the analytical asymptotic solution. 

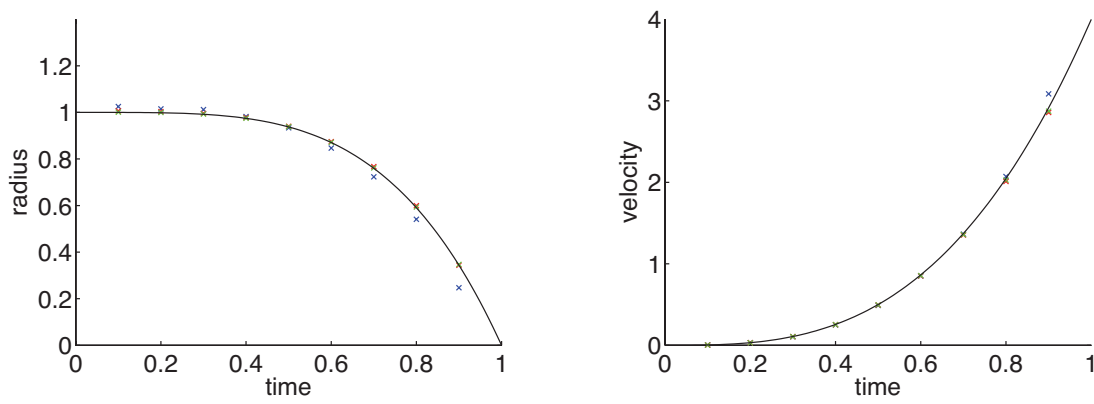

Fig. 24 Shell radius against time (left) and shell velocity against time (right). In these plots the black curve represents the exact solution $R(t)=1-t^{4}$ and $V(t)=4 t^{3}$, the blue x's represent the solution with no AMR, the red x's represent the solution with one level of AMR, and the green $x$ 's represent the solution with two AMR levels. We can see the convergence of the solution to the exact thin shell prediction upon increasing resolution.

\subsubsection{A More Realistic Implosion in the r-z Plane with Rayleigh-Taylor Instability Effects}

In this more challenging prototype problem perturbations are introduced into the initial geometric configuration of a finite thickness representation of a shell liner [8]. These perturbations are intended to generate vorticity due to Rayleigh-Taylor and/or Richtmyer-Meshkov type instabilities. Here the initial density profiles are given by a liner density of $\rho_{L}=1.0$, an exterior density of $\rho_{\text {exterior }}=0.025$, and a pre-fill density inside the linear of $\rho_{\text {pre-fill }}=0.05$. The initial pressure is constant with a value of $p_{0}=0.01$. The base computational grid has a mesh spacing of $h \approx 0.0025$ and uses two additional levels of factor 4 AMR to give an effective grid resolution of $h \approx 0.0015625$. In this case a linear current drive $I=\sqrt{12} t$ is employed.

Figure 25 shows a numerical Schlieren of the time evolution of the initial conditions where a sinusoidal perturbation to the initial interface has been introduced. This perturbation is intended to promote the growth of a particular unstable RaleighTaylor mode and thus create significant structure as the liner nears stagnation. Here the perturbation has amplitude 0.005 and period 0.125 and has been introduced along the inner boundary of the conducting shell. The initial pressure distribution, $p_{0}=0.01$, is selected to promote growth of R-T spikes ahead of the liner implosion at a sufficient rate so as to view their effect before stagnation. The instability evolves from the initial conditions on the right to the stagnation on axis on the left. The FCT AMR solution provides a very well resolved simulation of multiple unstable modes resulting in a complex pattern of R-T growth with complex interaction of shock waves at stagnation. It should be noted that the evolution of the R-T instability is qualitatively different from an actual Z-pinch system in which spikes lag behind the remaining liner material due the larger current flow in this contiguous material sheet (for example see [18, 23]). This is due to our simplified model 


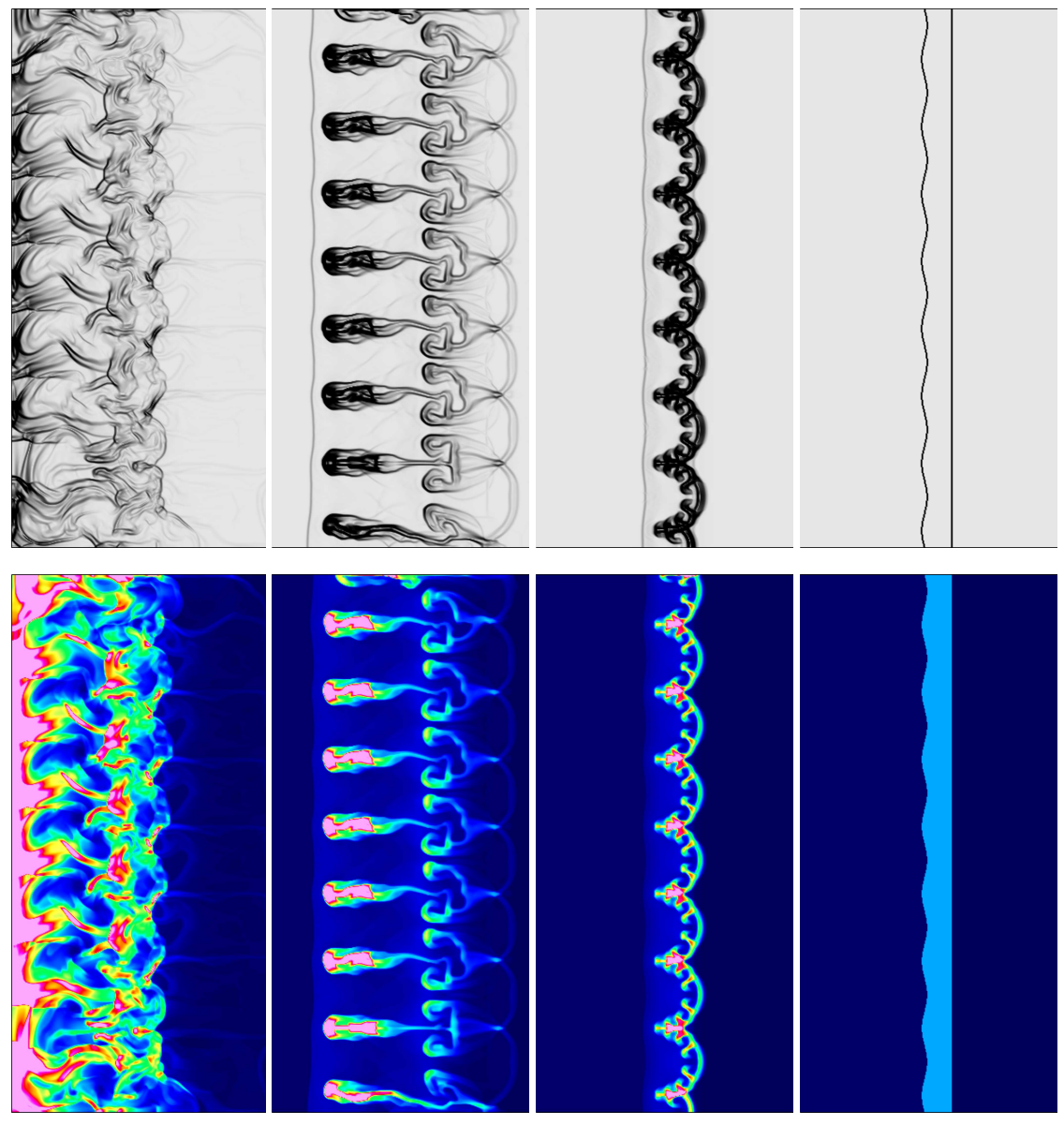

Fig. 25 A prototype Rayleigh-Taylor instability for a perturbed liner geometry for the magnetic implosion in $(r, z)$. An initial pressure distribution to promote growth of R-T spikes ahead of the liner implosion has been selected. The instability evolves from the initial conditions on the right to the stagnation on the axis on the left. The upper images are a numerical Schlieren and the lower images show the density at times (from left to right) $t=1.2, t=1.0, t=0.8$, and $t=0.0$.

assumption that defines the "current sheet" by the scalar $\lambda$ that cannot adequately model the preferential physical current flow through the contiguous liner material over the penetrating spikes. However the magnetic force term does produce spikes and sheets of material developed by the R-T effects and R-M instabilities as the strong-shock interacts with the trailing liner material sheet. These later stages have some qualitative similarities to actual Z-pinch implosions which gives indication to why such a simple testing procedure can be very beneficial in benchmarking the flow portion of simulation tools intended for shock-hydrodynamics applications. 


\subsubsection{An Idealized Z-pinch Implosion with Simplified Radiation Emission and a Self Convergence Study}

This final test prototype problem is intended to increase the complexity of the Zpinch prototype problem to include a phenomenological radiation emission model and to allow the evaluation of the FCT method by considering the estimated orderof-accuracy of the method in modeling the integrated radiation output from the implosion. In actual Z-pinch modeling efforts the simulation of the temporal characteristics of the radiation output is an important and challenging goal for these types of simulations. Even with the use of such a simplified model as described below, the results produced by the Euler system solver with the $\mathbf{J} \times \mathbf{B}$ source term model produce power pulses with qualitative similarities to experimental and full computational MHD results found in the literature (see e.g. Figure 9 in [25]).

One of the simplest radiation emission models one might consider is

$$
Q_{\text {rad }}=\sigma T^{4}
$$

where $T$ is the temperature obtained from the equation of state (2) and the caloric equation of state for an ideal gas as given by $\rho e=\rho C_{v} T$, where $C_{v}$ is the specific heat of the gas at constant volume. From [8]

$$
\sigma=\bar{\sigma} \rho \frac{1}{T^{2}}
$$

where $\bar{\sigma}$ is a constant chosen here to be $\bar{\sigma}=100.0$. The radiation emission is set to be active only in the liner material by multiplication with $\lambda$ so the final radiation source term becomes

$$
Q_{\text {rad }}=\bar{\sigma} \lambda \rho T^{2} .
$$

Obtaining resolved radiation profiles in an actual Z-pinch simulation is a tremendously challenging task due both to the complexity of the physically realistic radiation models as well as the mesh resolution requirements. With this difficulty in mind, this section presents two convergence studies where different aspects of this difficult regime are highlighted.

The first problem is a pinch in the $r-z$ plane with some small amount of pre-fill material and a stagnation point off axis at $r=0.1$ as for example on a rod. Here the pre-fill and exterior densities are $\rho_{\text {pre-fill }}=1 \times 10^{-3}, \rho_{\text {exterior }}=5 \times 10^{-4}$ and the initial pressure is $p=1 \times 10^{-5}$. A linear current drive $I=\sqrt{12} t$ is used so the current is rising as the material stagnates and radiates. The study is performed at a variety of uniform mesh resolutions corresponding to $h=1 / 160,1 / 320,1 / 640$, $1 / 1280,1 / 2560$ and $1 / 5120$ where $h$ is the grid spacing in the $r$ and $z$ directions. Figure 26 presents the radiation output and the total radiated power as a function of time for these resolutions and it is clear that the pulse is well behaved and largely converged at the finest resolution $h=1 / 5120$.

In order to gauge convergence, consider the convergence rate in two separate norms. The first and perhaps simplest is to look at is the extrapolated convergence 

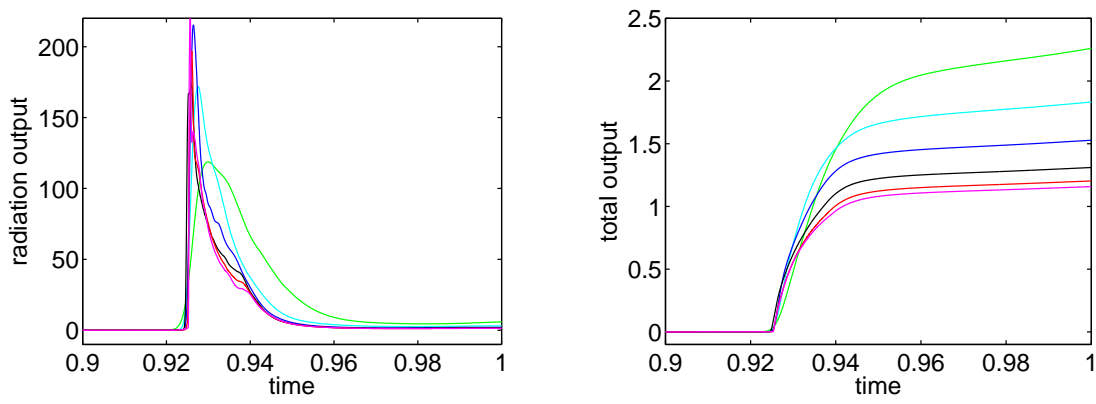

Fig. 26 Shown here are the radiation pulses (left) and total radiated power (right) obtained using the linear current model $I=\sqrt{12} t$ on a pinch with $\rho_{\text {pre-fill }}=1 \times 10^{-3}$ and a stagnation point at $r=0.1$. The simulation is carried out in the $r-z$ plane and the resolutions, from coarse to fine, are represented by green, cyan, blue, black, red and maroon.

rate as considered by Roy in [50]. In this analysis the final three data points for total radiated power from Table 10 are considered and an order of convergence is obtained, as in [50], from this data. For this data the convergence rate is judged to

Table 10 Total radiated power, peak power, and time to peak power for the radiation results presented in Figure 26. Here $N$ is a measure of grid resolution with $h=1 / N$.

\begin{tabular}{||c|c|c|c||}
\hline \hline$N$ & total radiated power & peak power & time to peak power \\
\hline 160 & 2.261 & 118.7 & .9299 \\
\hline 320 & 1.833 & 172.0 & .9267 \\
\hline 640 & 1.528 & 215.3 & .9264 \\
\hline 1280 & 1.311 & 174.0 & .9260 \\
\hline 2560 & 1.203 & 196.8 & .9261 \\
\hline 5120 & 1.158 & 227.8 & .9257 \\
\hline \hline
\end{tabular}

be $\kappa \approx 1.26$ for the total radiated power at $t=1.0$. For the second measure consider the self convergence in $L_{1}$ of individual radiation pulses to the finest computation $h=1 / 5120$ from Figure 26. Table 11 shows these results with a least squares fit to the convergence rate $\kappa \approx 1.08$. To put this result in context, for flows with solutions dominated by shocks a rate of $\kappa=O(\Delta x)$ is expected and for contact surface dominated flows a rate of $\kappa=O\left(\Delta x^{2 / 3}\right)$, for a second order method, is expected as described above. Finally it should be noted that because simulations are run at fixed CFL, spatial refinement and temporal refinement are carried out in a coordinated manner.

The second radiation result demonstrates stagnation with the very difficult prefill density set at $\rho_{\text {pre-fill }}=1 \times 10^{-6}$, the exterior density $\rho_{\text {exterior }}=5 \times 10^{-7}$ and stagnation on the center line. Here the power law current drive $I(t)=\sqrt{12\left(1-t^{6}\right) t^{4}}$ and $I=0$ for $t \geq 1$ is used rather than the linear drive. The softening of the problem 
Table 11 Self convergence results for the $L_{1}$ norm of the radiation pulses from Figure 26 as compared to the finest resolution $h=1 / 5120$. Here $\kappa \approx 1.08$ is a least squares fit for the convergence rate and $N$ is a measure of grid resolution with $h=1 / N$.

\begin{tabular}{||c|c||}
\hline \hline$N$ & self convergence error \\
\hline 160 & 2.614 \\
\hline 320 & 1.149 \\
\hline 640 & 0.5340 \\
\hline 1280 & 0.2672 \\
\hline 2560 & 0.1275 \\
\hline \hline$\kappa$ & 1.08 \\
\hline \hline
\end{tabular}

as a result of the more gentle current drive is more than made up for in the increased difficulty of resolving the stagnation on axis which makes numerical convergence extremely difficult. Simulations have been carried out for $h=1 / 160, h=1 / 320$, $1 / 640,1 / 1280,1 / 2560$ and 1/5120. Figure 27 presents the radiation output and
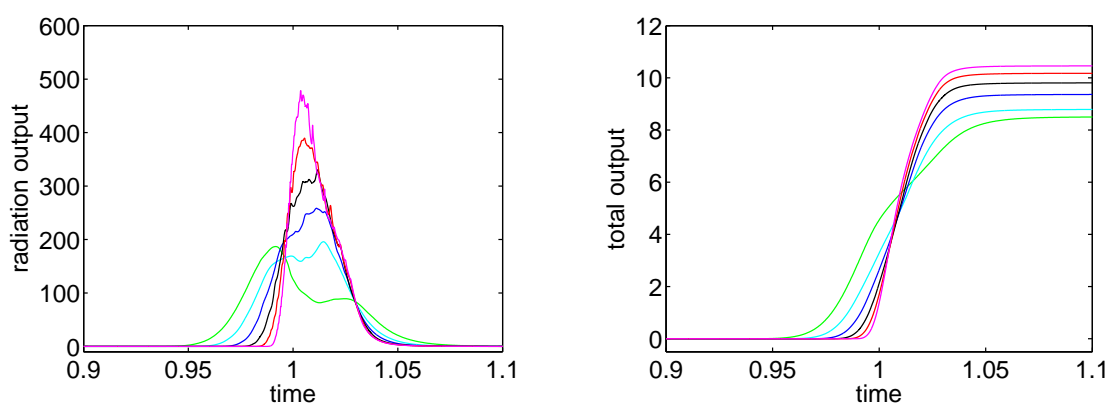

Fig. 27 Shown here are the radiation pulses obtained using the current model $I(t)=$ $\sqrt{12\left(1-t^{6}\right) t^{4}}$. The simulation is carried out in $(r, z)$ space with $r \in[0,1.25]$. The radiation output and total radiated power is plotted as a function of time with the simulations from coarse to fine represented by green, cyan, blue, black, red and maroon.

total radiated power as a function of time for these results and indicates strongly that convergence is near at hand for the finest resolution $h=1 / 5120$, but shows how difficult this problem is in terms of sufficient resolution for convergence. Indeed some aspects of the simulation seem to be well resolved such as the peak output location as a function of time, but even at this fine resolution the peak power is clearly not well resolved.

As before convergence is measured in two ways. The first takes the extrapolated convergence rate for the total radiated power at $t=1.0$ in Table 12 as in [50]. The result here is $\kappa \approx 0.37$ which is obviously not excellent, but still convergent and for such a difficult problem still considered reasonable. The second measure judges self convergence of individual radiation profiles in Figure 27 using the $L_{1}$ norm. 
Table 12 Total radiated power, peak power, and time to peak power for the radiation results presented in Figure 27. Here $N$ is a measure of grid resolution with $h=1 / N$.

\begin{tabular}{||c|c|c|c||}
\hline \hline$N$ & total radiated power & peak power & time to peak power \\
\hline 160 & 8.500 & 186.9 & 1.015 \\
\hline 320 & 8.790 & 195.9 & 1.011 \\
\hline 640 & 9.365 & 258.5 & 1.011 \\
\hline 1280 & 9.808 & 330.4 & 1.012 \\
\hline 2560 & 10.18 & 389.7 & 1.006 \\
\hline 5120 & 10.46 & 478.5 & 1.003 \\
\hline \hline
\end{tabular}

Table 13 shows these results and demonstrates that even here the convergence rate is $\kappa \approx 0.75$ which is quite good.

Table 13 Self convergence results for the $L_{1}$ norm of the radiation pulses from figure 27 as compared to the finest resolution $h=1 / 5120$. Here $\kappa \approx 0.75$ is a least squares fit for the convergence rate and $N$ is a measure of grid resolution with $h=1 / N$.

\begin{tabular}{||c|c||}
\hline \hline$N$ & self convergence error \\
\hline 160 & 10.74 \\
\hline 320 & 6.920 \\
\hline 640 & 4.450 \\
\hline 1280 & 2.654 \\
\hline 2560 & 1.290 \\
\hline$\kappa$ & 0.75 \\
\hline \hline
\end{tabular}

\section{Conclusions}

This chapter has outlined the development and assessment of a high-resolution FCT algorithm for the Euler equations on structured overlapping grids as in [6]. The implementation of the FCT method for overlapping grids was based on the Overture framework and included modifications and extensions to the classical FCT algorithm. These extensions included the modifications required for the discretization on curvilinear grids as well as the inclusion of a Jameson-style fourth-order artificial viscosity to remove the high frequency noise produced by FCT. Improvements were made to the FCT algorithm to eliminate entropy violating shocks that can occur at sonic points in rarefaction waves. Difficulties occurring in strong rarefaction waves, where a near vacuum state is produced when the gas separates at velocities greater than the sound speed, were also addressed. In addition we discussed FCT for moving overlapping grids. 
We have evaluated this new FCT method on a series of benchmark high-speed flow problems and compared the results to those obtained using a high-resolution Godunov method. This investigation confirmed the expected convergence character for manufactured solutions as well as isolated contact and shock waves. The overlapping grid capabilities were used to study the interaction of a planar shock by fixed and movable rigid cylinders as well as irregular Mach reflection of a strong shock on an inclined ramp. Where possible the FCT method was compared to a well characterized Godunov method. Overall the results obtained by the FCT and Godunov methods were found to be very similar. The FCT solutions tended to have a somewhat higher resolving capability but also to contain more numerical noise. It should be noted that our implementation of the FCT method was quite costly in comparison to the Godunov method. This is due to the large number of characteristic transformations, the smaller time step required, and the apparent difficulty in removing high frequency noise which tends to flag cells for refinement by the AMR algorithm [6]. Recent developments in alternate limiting procedures for conserved and primitive variables, as well as linearized FCT algorithms for implicit calculations might help to reduce the overhead of the FCT algorithm [40]. Finally, the extensive comparisons and order-of-accuracy results presented in this study suggest that the FCT method may be a viable option for cases where Riemann solvers are expensive or not readily known, or for cases with extremely large jumps where more traditional methods may have difficulty. This type of challenging problem was illustrated with the inclusion of the prototype Z-pinch implosion as discussed in [8].

\section{References}

1. Arora, M., Roe, P.L.: On postshock oscillations due to shock capturing schemes in unsteady flows. J. Comput. Phys. 130, 25-40 (1997)

2. Ascher, U.M., Petzold, L.R.: Computer Methods for Ordinary Differential Equations and Differential-Algebraic Equations. SIAM, Philadelphia (1998)

3. Baker, T.: Mesh generation for the computation of flowfields over complex aerodynamic shapes. Computers Math. Applic. 24, 103-127 (1992)

4. Banks, J.W., Aslam, T., Rider, W.J.: On sub-linear convergence for linearly degenerate waves in capturing schemes. J. Comput. Phys. 227(14), 6985-7002 (2008)

5. Banks, J.W., Henshaw, W.D., Schwendeman, D.W., Kapila, A.K.: A study of detonation propagation and diffraction with compliant confinement. Combust. Theory and Modelling 12(4), 769-808 (2008)

6. Banks, J.W., Henshaw, W.D., Shadid, J.N.: An evaluation of the FCT method for high-speed flows on structured overlapping grids. J. Comput. Phys. 228(15), 5349-5369 (2009)

7. Banks, J.W., Schwendeman, D.W., Kapila, A.K., Henshaw, W.D.: A high-resolution Godunov method for compressible multi-material flow on overlapping grids. J. Comput. Phys. 223, 262-297 (2007)

8. Banks, J.W., Shadid, J.N.: An Euler system source term that develops prototype z-pinch implosions intended for the evaluation of shock-hydro methods. Int. J. Numer. Meth. Fluids 61, 725-751 (2009))

9. Bellan, P.: Miniconference on astrophysical jets. Physics of Plasmas 12, 058,301 (2005)

10. Berger, M.J., Oliger, J.: Adaptive mesh refinemenr for hyperbolic partial differential equations. J. Comput. Phys. 53, 484-512 (1984) 
11. Boris, J.P., Book, D.L.: Flux-corrected transport. I. SHASTA, a fluid transport algorithm that works. J. Comput. Phys. 11, 38-69 (1973)

12. Boris, J.P., Book, D.L.: Flux-corrected transport III. Minimal-error FCT algorithms. J. Comput. Phys. 20, 397-431 (1976)

13. Boris, J.P., Book, D.L., Hain, K.: Flux-corrected transport II: Generalizations of the method. J. Comput. Phys. 18, 248-283 (1975)

14. C. Richard DeVore: Flux-corrected transport techniques for multidimensional compressible magnetohydrodynamics. J. Comput. Phys. 92, 142-160 (1991)

15. Cerqueira, A.H., m. De Gouveia Dal Pino, E.: MHD numerical simulations of proto-stellar jets. Space Science Reviews 107, 337-340 (2003)

16. Chan, W.: A unified overset grid generation graphical interface and new concepts on automatic gridding around surface discontinuities. In: Proceedings of the 4th Symposium on Overset Composite Grid and Solution Technology (1998)

17. Chesshire, G., Henshaw, W.: Composite overlapping meshes for the solution of partial differential equations. J. Comput. Phys. 90, 1-64 (1990)

18. Chittenden, J., Lebedev, S., Bland, S., Beg, F., Haines, M.: One-, two-, and three-dimensional modeling of the different phases of wire array z-pinch evolution. Physics of Plasmas 8(5), 2305-2314 (2001)

19. Colella, P., Woodward, P.R.: The piecewise parabolic method (PPM) for gas-dynamical simulations. J. Comput. Phys. 54(1), 174-201 (1984)

20. Dumbser, M., Moschetta, J.M., Gressier, J.: A matrix stability analysis of the carbuncle phenomenon. J. Comput. Phys. 197, 647-670 (2004)

21. Dyke, M.V.: An Album of Fluid Motion. The Parabolic Press, Stanford, California (1982)

22. Foster et. al.: High-energy-density laboratory astrophysics studies of jets and bow shocks. The Astrophysical Journal 634L, 77-80 (2005)

23. Garasi, C.J., Bliss, D.E., Mehlhorn, T.A., Oliver, B.V., Robinson, A.C., Sarkisov, G.S.: Multidimensional high energy density physics modeling and simulation of wire array Z-pinch physics. Physics of Plasmas 11(5), 2729-2737 (2003)

24. Greenough, J.A., Rider, W.J.: A quantitative comparison of numerical methods for the compressible euler equations: fifth-order WENO and piecewise-linear godunov. J. Comput. Phys. 196, 259-281 (2004)

25. Haines, M., lebedev, S., Chittenden, J., Beg, F., Bland, S., Dangor, A.: The past, present and future of Z pinches. Physics of Plasmas 7(5), 1672-1680 (2000)

26. Harten, A.: The artificial compression method for computation of shocks and contact discontinuities. I. single conservation laws. Commun. Pur. Appl. Math. 30(5), 611-638 (1977)

27. Harten, A., Lax, P.D., van Leer, B.: On upstream differencing and Godunov type schemes for hyperbolic conservation laws. SIAM Rev. 25, 33-61 (1983)

28. Hedstrom, G.W.: The rate of convergence of some difference schemes. J. SIAM Numer. Anal. 5(2), 363-406 (1968)

29. Henshaw, W.: Mappings for Overture, a description of the Mapping class and documentation for many useful Mappings. Research Report UCRL-MA-132239, Lawrence Livermore National Laboratory (1998)

30. Henshaw, W.D.: OverBlown: A fluid flow solver for overlapping grids, reference guide. Research Report UCRL-MA-134289, Lawrence Livermore National Laboratory (1999)

31. Henshaw, W.D., Schwendeman, D.W.: An adaptive numerical scheme for high-speed reactive flow on overlapping grids. J. Comput. Phys. 191(2), 420-447 (2003)

32. Henshaw, W.D., Schwendeman, D.W.: Moving overlapping grids with adaptive mesh refinement for high-speed flow. J. Comput. Phys. 216(2), 744-779 (2006)

33. Henshaw, W.D., Schwendeman, D.W.: Parallel computation of three-dimensional flows using overlapping grids with adaptive mesh refinement. J. Comput. Phys. 227(16), 7469-7502 (2008)

34. Jameson, A.: Transonic flow calculations for aircraft. In: Numerical Methods in Fluid Dynamics, vol. 1127 of Lecture Notes in Mathematics, pp. 156-242. Springer-Verlag (1983) 
35. Jameson, A., Schmidt, W., Turkel, E.: Numerical solution of the euler equations by finite volume methods using runge-kutta time-stepping schemes. In: AIAA 14th Fluid and Plasma Dynamic Conference (1981)

36. Jiang, G.S., Shu, C.W.: Efficient implementation of weighted eno schemes. J. Comput. Phys. 126(1), 202-228 (1996)

37. Kapila, A.K., Schwendeman, D.W., Bdzil, J.B., Henshaw, W.D.: A study of detonation diffraction in the Ignition-and-Growth model. Combust. Theory and Modelling (to appear)

38. Karni, S., Čanić, S.: Computations of slowly moving shocks. J. Comput. Phys. 136, 132-139 (1997)

39. Kuzmin, D., Löhner, R., Turek, S. (eds.): Flux-Corrected Transport. Springer (2005)

40. Kuzmin, D., Löhner, R., Turek, S. (eds.): Flux-Corrected Transport, Second Edition: AFC II. Springer (2011)

41. LeVeque, R.J.: Numerical Methods for Conservation Laws. Birkhauser, Basel (1992)

42. Liberman, M.A., Groot, J.S.D., Toor, A., Spielman, R.B.: Physics of high-density Z-pinch plasmas, pp. 7-10,19-28,44-54,133-163,239-243. Springer Verlag, New York (1999)

43. M. K. Matzen et. al.: Pulsed-power-driven high energy density physics and inertial confinement fusion research. Physics of Plasmas 12, 055,503 (2005)

44. Matzen, M.K.: Z pinches as intense x-ray sources for high-energy density physics applications. Physics of Plasmas 4(5), 1519-1527 (1997)

45. National Research Council National Academies: Frontiers in high energy density physics: The X-games of contemporary science, pp. 18-19,34-119. Springer Verlag, National Academies Press (2003)

46. Petersson, N.A.: Hole-cutting for three-dimensional overlapping grids. SIAM J. Sci. Comp. 21, 646-665 (1999)

47. Quirk, J.J.: A contribution to the great riemann solver debate. Int. J. Numer. Meth. Fl. 18, 555-574 (1994)

48. Rider, W.J., Greenough, J.A., Kamm, J.R.: Accurate monotonicity- and extrema-preserving methods through adaptive nonlinear hybridizations. J. Comput. Phys. 225, 1827-1848 (2007)

49. Roe, P.L.: Approximate Riemann solvers, parameter vectors, and difference schemes. J. Comput. Phys. 43, 357-372 (1981)

50. Roy, C.J.: Grid convergence error analysis for mixed-order numerical schemes. AIAA Journal 41(4), 595-604 (2003)

51. S. V. Lebedev et. al.: Laboratory astrophysics and collimated stellar outflows: The production of radiatively cooled hypersonic plasma jets. The Astrophysical Journal 564, 113-119 (2002)

52. Sod, G.A.: A survey of several finite difference methods for systems of nonlinear hyperbolic conservation laws. J. Comput. Phys. 27(1), 1-31 (1978)

53. Stygar, W.A., Ives, H.C., Fehl, D.L., Cuneo, M.E., Mazarakis, M.G., Bailey, J.E., Bennett, G.R., Bliss, D.E., Chandler, G.A., Leeper, R.J., Matzen, M.K., McDaniel, D.H., McGurn, J.S., McKenney, J.L., Mix, L.P., Muron, D.J., Porter, J.L., Ramirez, J.J., Ruggles, L.E., Seamen, J.F., Simpson, W.W., Speas, C.S., Spielman, R.B., Struve, K.W., Torres, J.A., Vesey, R.A., Wagoner, T.C.: X-ray emission from $z$ pinches at $10^{7}$ A: Current scaling, gap closure, and shotto-shot fluctuations. Phys. Rev. E 69(4), 046,403 (2004). DOI 10.1103/PhysRevE.69.046403

54. T.A. Gardiner et. al.: MHD models and laboratory experiments of jets. Astrophysics and Space Science 287, 69-74 (2003)

55. Toro, E.F.: Riemann Solvers and Numerical Methods for Fluid Dynamics. Springer, Berlin (1999)

56. Tóth, G., Odstrcil, D.: Comparison of some flux corrected transport and total variation diminishing numerical schemes for hydrodynamic and magnetohydrodynamic problems. J. Comput. Phys. 128(1), 82-100 (1996)

57. Whitham, G.B.: Linear and Nonlinear Waves. Wiley-Interscience, New York (1974)

58. Woodward, P., Colella, P.: The numerical simulation of two-dimensional fluid flow with strong shocks. J. Comput. Phys. 54, 115-173 (1984)

59. Zalesak, S.T.: Fully multidimensional flux-corrected transport algorithms for fluids. J. Comput. Phys. 31, 335-362 (1979)

60. Zalesak, S.T.: The design of flux-corrected transport (FCT) algorithms on structured grids. Ph.D. thesis, George Mason University (2005) 